\title{
Tracking Attentional States: Assessing the Relationship Between Sustained and Selective Focused Attention in Visual Working Memory
}

\author{
Andra Arnicane $^{1}$ and Alessandra S. Souza ${ }^{1,2}$ \\ ${ }^{1}$ University of Zurich; ${ }^{2}$ University of Porto
}

This version of the article has been accepted for publication in Attention, Perception \& Psychophysics, after peer review and is subject to Springer Nature's AM terms of use, but is not the Version of Record and does not reflect post-acceptance improvements, or any corrections. The Version of Record is available online at: http://dx.doi.org/10.3758/s13414-021-02394-y

\section{Author Note}

Andra Arnicane, Orcid: https://orcid.org/0000-0002-7304-3381

Alessandra S. Souza, Orcid: https://orcid.org/0000-0002-1057-8426

We have no conflict of interest to disclose. This research was supported by a grant from the Velux Foundation to A. S. Souza (project $\mathrm{n}^{\circ}$ 1053). This research was presented on the Virtual Working Memory Symposium 2020, as a poster at the virtual $61^{\text {st }}$ Annual Meeting of the Psychonomic Society, and is shared as a poster on ResearchGate. Correspondence concerning this article should be addressed to Andra Arnicane, Department of Psychology, University of Zurich, Binzmühlestrasse 14, CH-8050, Zürich, Switzerland. E-mail: arnicanea@gmail.com. The study materials and data are available at Open Science Framework: https://osf.io/z2dpc/ 


\begin{abstract}
Attention has multiple influences on visual working memory (VWM). Fluctuations in sustained attention predict VWM performance. Furthermore, focusing selective attention in VWM by retro-cuing the to-be-tested item during maintenance, boosts retrieval. So far, we lack knowledge how the ability to focus selective attention relates to the state of sustained attention during the VWM trial. Here, we combined a retro-cue task and a self-rated attention protocol to test whether focusing selective attention via retro-cues: (1) mitigates spontaneous attention fluctuations, in which case retro-cues should be more helpful under low levels of self-rated attention; (2) depends on optimal state of sustained attention, in which case retro-cue benefits should be largest under high levels of self-rated attention; or (3) is independent of sustained attention, in which case retro-cue benefits and self-rated attention effects should be additive. Our data supported the additive hypothesis. Across four experiments, self-rated attention levels predicted continuous reproduction of colors. Retro-cue trials produced better recall and higher rated attention. Critically, retro-cues improved recall to a similar extent across all levels of self-rated attention. This indicates that attention has multi-faceted and independent contributions to VWM.
\end{abstract}

Keywords: Visual Working Memory, Attention, Retro-Cue, Self-Report, continuous reproduction task 


\section{Tracking Attentional States: Assessing the Relationship Between Sustained and Selective Focused Attention in Visual Working Memory}

Visual Working memory (VWM) serves ongoing cognition by maintaining and processing visual information across short periods of time. The efficiency of VWM is severely constrained by its capacity, often quantified as the number of items that can be concurrently held in mind. Healthy younger adults can maintain, on average, four visual representations accessible in VWM. This estimate, however, is subject to variation between individuals (Cowan, 2010; Luck \& Vogel, 2013) and also within-individuals, reflecting trial-by-trial fluctuations due to changes in sustained attention (Adam et al., 2015; Adam \& Vogel, 2017; deBettencourt et al., 2019, 2020; Fukuda et al., 2015; Mrazek et al., 2012; Rademaker et al., 2012). The contribution of attentional fluctuations to VWM performance has been examined, for example, by asking participants to rate their attentional levels towards the task (Adam \& Vogel, 2017; Unsworth \& Robison, 2016) or by interspersing memory probes in a sustained attention task at moments associated with high and low levels of sustained attention (deBettencourt et al., 2018, 2019). This has showed that memory performance is highly related to overall levels of attentiveness.

Relevance of attention for VWM performance is also evident from the literature showing that retrospective cueing of selective attention towards a specific representation during maintenance (with so-called retro-cues) improves recall. Since the first seminal studies (Griffin \& Nobre, 2003; Landman et al., 2003), ample research has confirmed the 
robustness of the attentional benefit elicited by retro-cues for memory accuracy as well as response times (see Myers et al., 2017; Souza \& Oberauer, 2016 for reviews).

In summary, on the one hand, fluctuations of sustained attention predict how much information has entered VWM, whereas, on the other hand, selective focused attention can strengthen the focused representation, boosting retrieval. So far, it is unclear how sustained attention and selective attention relate to each other in VWM: do they represent a single source of variation in memory performance, or do they contribute independently to it? Attention is not a monolithic concept (Chun et al., 2011), however, theories have not considered the roles that different types of attention play in the working memory system (Oberauer, 2019), and researchers have not considered the joint contribution of these factors to memory performance. Here we ask whether the benefit of retro-cueing a VWM representation is related to the overall level of sustained attention towards the memory task. In the following sections, we discuss the literature on sustained attention and selective attention in VWM, and the metacognitive awareness regarding these states. Finally, we present our hypotheses and our approach to answer our questions.

\section{VWM and Attention Fluctuations}

Attention naturally fluctuates within and across trials (Esterman \& Rothlein, 2019). These fluctuations can be tracked via subjective self-reports as well as objective task performance measures. The ability to sustain attention across trials is often measured with the Sustained Attention to Response Task (SART, Robertson et al., 1997). In this task, participants respond to frequent stimuli and must withhold responses for rare targets. Errors in this task are often linked to self-reported decreases in task focus, aka 
mind wandering (see Randall et al., 2014; Smallwood \& Schooler, 2006 for reviews). Recent research has shown that lapses of attention and VWM are related: By tracking response time variability in a hybrid SART and VWM task, deBettencourt et al. (2019) showed that VWM probes presented when participants were inferred to be attentive or inattentive produced high and low levels of VWM performance. Other studies reported that VWM accuracy fluctuates in concert with self-rated attention and confidence (Adam \& Vogel, 2017; Arnicane et al., 2021), and reports of on-task focus versus mindwandering (Adam \& Vogel, 2017; Unsworth \& Robison, 2016). Similarly, trial-to-trial fluctuations in attentiveness strongly correlate with performance in other WM tasks, for example in the $n$-back (Rummel \& Boywitt, 2014) and complex span (Mrazek et al., 2012).

The idea that VWM performance is supported by sustained attention is implemented in several computational models of VWM capacity that include attentional fluctuations as a parameter to capture trial-by-trial variations in performance (Adam et al., 2015; Rouder et al., 2008). An instantiation of one such model used a binary fullattention vs. no-attention (i.e., attention lapse) parameter to adjust model's behavior on a trial-by-trial basis (Rouder et al., 2008). According to this implementation, sudden lapses of attention may explain why even individuals with high VWM capacity sometimes perform very poorly, or why people may fail on trials with within-capacity set sizes (e.g., having only one or two items). Further work compared a binary lapse model to a one with graded attention and found that the latter more fully accounted for the data (Adam et al., 2015; see also Killebrew et al., 2018). Together, these empirical findings and modelling 
approaches illustrate that the waxing and waning of sustained attention plays a

considerable role in VWM performance.

\section{VWM and Selective Attention}

A vital selection device supporting VWM performance is the focus of attention: a mechanism for selective prioritization of one or several items held in VWM (Cowan, 2011; Oberauer, 2009, 2013). Directing attention to VWM contents - i.e., by bringing them into the focus of attention - renders representations more durable by strengthening the items' bindings to the context such as their spatial location in the visual array (Oberauer, 2013). This increases the item's resistance to interference from switches of visual and central attention - namely, when being presented with a secondary task before the memory test (Hollingworth \& Maxcey-Richard, 2013; Makovski \& Jiang, 2007; Makovski \& Pertzov, 2015; Matsukura et al., 2007; Rerko et al., 2014; Sligte et al., 2008; van Moorselaar, Gunseli, et al., 2015), and decreases items' susceptibility to the interference by decision-making processes and the test display itself (Gunseli et al., 2018; Makovski et al., 2008, 2010; Rerko et al., 2014; Souza et al., 2016; Tabi et al., 2019; van Moorselaar, Gunseli, et al., 2015). Furthermore, repeated focusing on visual representations boosts memory in a cumulative fashion, which results in better memory for items that have been attended several times compared to those attended once (Rerko \& Oberauer, 2013; Souza, Vergauwe, et al., 2018; Souza \& Oberauer, 2017).

\section{Meta-Cognition About Attentional States}

Subjective experience can be assessed by soliciting self-reports. To date, VWM studies have asked participants to rate either their attentional state or their response confidence. Introspection on attentional states is assumed to reflect knowledge about the 
state of preparedness to perform the task or of task focus more generally (Adam \& Vogel, 2017; Unsworth \& McMillan, 2014a, 2014b). Confidence ratings, conversely, request participants to predict or evaluate their performance in the memory test, which is assumed to be guided by the precision of the relevant memory representations (Mayer et al., 2020; Peters et al., 2019; Rademaker et al., 2012; Samaha \& Postle, 2017; van den Berg et al., 2017; Vandenbroucke et al., 2014). Both attention and confidence reports have been found to relate to performance in VWM tasks. Although attentional reports do not require performance evaluation, it can be difficult to disentangle to what extent performance evaluation affects these reports. Even though our main goal in this study was to collect attention reports, we will review results of both types of studies as they provide complementary information about the reliability of subjective reports, and how attention focusing modulates VWM performance.

Only a handful of studies have collected attentional reports in working memory and VWM tasks. Mrazek et al. (2012) found that self-reported attention (rated on a 1-5 Likert scale) predicted individual differences in complex span and reasoning tasks. Moreover, trial-by-trial analyses revealed attentional lapses, such that lower rated attention was related to poorer performance on that particular trial. Adam and Vogel (2017) asked for participants' ratings of attention on a Likert scale ranging from 1-9 in one experiment and response confidence using a binary measure (confident vs. not confident) in another experiment during whole-report VWM tasks. Both types of ratings covaried with the number of correctly reported items on a trial-by-trial basis. This demonstrates that both memory performance and attention levels fluctuate across time, 
and that people can introspect on their attentional states. Similar results were observed by Arnicane et al. (2021) in a sample of younger and older adults.

Confidence ratings have been more broadly used in VWM research. Introspective confidence judgements track memory accuracy when recall is measured on a continuous scale (Mitchell et al., 2018; Peters et al., 2019; Rademaker et al., 2012), as well as in change detection (Berryhill et al., 2012; Mayer et al., 2020; Sahar et al., 2020). This indicates that trial-to-trial fluctuations in VWM for continuous features are accessible to introspection similar to memory for discrete colors (Adam \& Vogel, 2017; Arnicane et al., 2021; Peters et al., 2019).

In a change-detection task, Berryhill et al. (2012) presented informative spatial retro-cues or uninformative neutral cues and solicited confidence ratings after every trial. They found that retro-cueing increased both performance accuracy and response confidence, leading them to conclude that the pattern of confidence ratings reflected participants' awareness of the retro-cue benefit for their performance (see also Vandenbroucke et al., 2014). However, confidence ratings were significantly higher after retro-cues also on trials with inaccurate responses. This is not surprising, as peoples' metacognitive insight into the contents of their VWM is imperfect (Adam \& Vogel, 2017). Hence, a subset of retro-cued trials with inaccurate responses could have received high confidence ratings. Another possibility is that, via the confidence rating, participants expressed their subjective experience of attention. This may indicate that selective focusing attention on a memory item increases peoples' overall level of attention. 


\section{The Present Study}

In sum, studies on the role of sustained-attention as reflected in self-reports of attentional state, and studies on the retro-cue effect have demonstrated two ways in which attention and VWM are interwoven: On one hand, fluctuations and lapses of sustained attention hinder VWM performance (Adam \& Vogel, 2017; deBettencourt et al., 2018, 2019) and, on the other hand, selective focusing via retro-cues benefits VWM accuracy (Souza \& Oberauer, 2016). Attention reports show that participants are aware of the wax and wane of their sustained attention towards the task (Adam \& Vogel, 2017; Mrazek et al., 2012) and these reports track VWM performance similar to confidence ratings (Berryhill et al., 2012; Rademaker et al., 2012; Vandenbroucke et al., 2014). However, it is not yet understood whether the retro-cue benefit depends on the overall level of sustained attention.

The aim of the present study is to leverage the information from attention selfreports to uncover the interplay of sustained and selective attention in VWM. We combined a VWM task requiring maintenance of continuously varying colors with a retro-cue paradigm and an attention report protocol. Attention reports were requested on a small subset of trials $(20 \%)$ both when participants were maintaining the whole memory set with no information of which item would be tested (assumed to only engage sustained distributed attention), and after a retro-cue had indicated the to-be-tested item (assumed to engage selective focused attention).

Previous studies showed that attention reports were positively associated with accuracy in a discrete whole-report VWM task (Adam \& Vogel, 2017; Arnicane et al., 2021). Here, we aimed to extend this finding by testing one item per trial in a continuous 
color reproduction task, aka delayed estimation task (Prinzmetal et al., 1998; Wilken \& Ma, 2004; Zhang \& Luck, 2008), which provides a sensitive measure of memory accuracy. In this type of task, participants reproduce the feature (in our case color) of the tested item on a continuous scale (here a color wheel). The error in reporting the color of a probed item increases as the memory load in the task increases (Hardman et al., 2017; Mitchell et al., 2018; Zhang \& Luck, 2008), and this error is reduced when the testrelevant item is cued during maintenance (Souza, Rerko, Lin, et al., 2014). Assessing the relation between attention reports and recall error in the no-cue vs. retro-cue conditions will help to shed light on how selective focused attention interacts with states of sustained attention.

The primary goal of our study was to assess the covariation between attention reports and VWM performance, aiming to distinguish between three hypotheses about the interplay of sustained and focused attention in VWM. The first possibility is that retrocue benefit is conditional on participants' optimal state of sustained attention because only then participants would have enough information about the memory array in mind. This implies that the cueing benefit on accuracy would only emerge when attention was rated as high (see Figure 1A). The second possibility is that the opposite pattern materializes: Retrospective focusing is more beneficial when attention has fluctuated beforehand (as reflected in lower levels of self-rated attention), whereas at already high levels of attention the cue boost is smaller (Figure 1B). This pattern would be consistent with the idea that focused attention can rescue people from a state of mind-wandering. Lastly, cueing could increase accuracy by a steady increment regardless of self-rated 
attentional level, indicating separate contributions of sustained attention and selective focused attention to VWM performance.

\section{Figure 1}

Hypothetical Scenarios for the Interplay of Sustained Attention and Selective Focused Attention Effects on VWM Performance
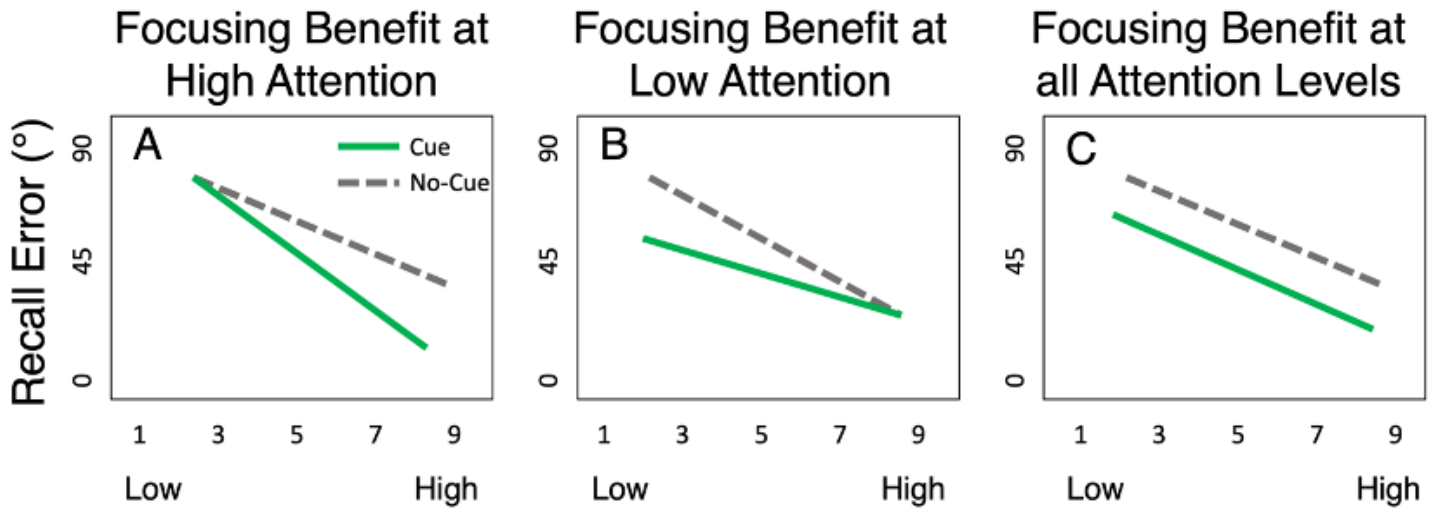

Attention Rating

Note. The figure depicts hypothetical levels of error in reproducing colors from memory (with better performance indicated by lower values) as a function of self-rated attention $(x$-axis) and whether there was the presentation or not of a retro-cue during the retention interval (Cue vs. No-Cue). Panel A visualizes the scenario in which retro-cue benefits are larger, the higher the level of self-rated attention, whereas Panel B illustrates the opposing pattern. These two scenarios are consistent with interdependence between sustained and selective attention. Panel $\mathrm{C}$ visualizes the scenario of independent, additive contributions of self-rated attention and the retro-cue benefit to VWM performance.

\section{Experiment 1}

Our main goal was to assess the interplay of sustained and selective focused attention in VWM. Attention ratings (on a scale of 1-9) were requested in $20 \%$ of the trials. We operationalized sustained attention with no-cue trials, and focused attention with trials presenting informative retro-cues, and examined the joint contribution these two types of attention on recall (as illustrated in Figure 1). Our main interest pertained to how the size of the retro-cue benefit varies as a function of self-rated attention levels. 
A secondary question we aimed to address here is whether retro-cues could also alter the subjective experience of attention, as reflected in a main effect of retro-cueing on attention reports. In other words, retro-cues could modulate not only the deployment of selective attention towards the cued item, but also the experience of attention participants report. For example, the retro-cue could increase the state of alertness of the participants, leading to higher levels of self-rated attention. However, several features of the retro-cue procedure may account for this effect. Increased self-rated attention following a retro-cue may indicate that participants assigned a higher share of attention towards the cued item. However, because the retro-cue presents visual information during maintenance, it stands to question whether the sudden onset of a visual event increases arousal, which also may increase self-rated attention, even if this stimulus does not allow one to focus attention. To control for this alternative explanation, a neutral-cue baseline presented an uninformative visual stimulus, matched in timing to the retro-cue appearance. The arousal hypothesis predicts that self-rated attention should increase following both retro-cues and neutral-cues. Another possibility is that the retro-cue prevents attention from receding over time. To address this, we included two no-cue baseline conditions that varied the retention interval: a no-cue short baseline (matched in retention interval to the time until the retro-cue onset), and a no-cue long baseline (matched to the total trial-time in the neutral- and retro-cue trials). If retro-cues protect attention from dropping over time, their effect on attention ratings should emerge only compared to the long, but not to the short baseline.

\section{Method}

\section{Participants}


Based on previous retro-cue studies in our lab, we defined a target sample size of $N=20$. Twenty students from Zurich universities $\left(M_{\text {Age }}=25.65, S D=4.12,16\right.$ women $)$ completed two sessions (ca. 1 hour each) and were compensated with $30 \mathrm{CHF}$ or course credit. For all experiments reported here, participants self-reported normal color vision. Written informed consent was obtained from all participants before the experiment, and participants were debriefed regarding the purpose of the study at the end. The study protocol is in line with the ethical guidelines of the institutional review board as established by the completion of a self-assessment checklist prior to data collection (checklist can be found here: https://www.phil.uzh.ch/de/forschung/ethik.html) and it did not require specific approval.

\section{Procedure}

The experiment was programmed in MATLAB (The MathWorks, Natick, MA) using the Psychophysics Toolbox 3 (Brainard, 1997; Pelli, 1997). All materials, data, and analysis scripts are available at Open Science Framework (https://osf.io/z2dpc/?view_only=bda94be6c4994dbfa736e6f57a464fa5).

The task was to memorize five colored discs. Figure 2 illustrates the flow of events in the task. At the beginning of a trial, a white fixation cross ( 1 ize $=40$ pixels) appeared for $500 \mathrm{~ms}$ against a grey background (RGB: 128, 128, 128). Next, five colored discs (radius $=35$ pixels) arranged equidistantly along an imaginary ring (radius $=200$ pixels) were shown for $500 \mathrm{~ms}$. The discs' colors on each trial were randomly sampled (without replacement) from 360 continuous color values defined in the CIE L*a*b color space $(L=70, a=20, b=38$, and radius $=60)$, and there was no minimal distance constraint between the colors. The positions of the discs remained constant throughout 
the experiment. Offset of the memory array was followed by a 1-s blank retention interval. In the no-cue short baseline condition, this 1-s retention interval was followed directly by the memory test. In the retro-cue condition, after the 1-s retention interval, a white arrow (radius $=80$ pixels) pointing to the location of the to-be-tested memory item was drawn in the center of the screen for $250 \mathrm{~ms}$, followed by another 1-s blank interval. The neutral-cue condition was identical to the retro-cue condition, except that five arrows were drawn simultaneously, pointing to all item locations at once. In the no-cue long baseline condition, the blank retention interval continued for $2.25 \mathrm{~s}$, thereby equating the trial duration to the retro- and neutral-cue conditions. At test, a black circle was drawn at one of the positions of the memory array, indicating the probed item, together with a color wheel (radius $=540$ pixels, randomly rotated on each trial). A white circle $($ size $=$ 30 pixels) was drawn around the current position of the mouse when it was moved on the color wheel, and the probe item's color changed according to the mouse position on the wheel. To respond, participants clicked the left mouse button. The inter-trial interval comprised $500 \mathrm{~ms}$. After every quarter of the trials, the experiment paused for a selfpaced break and participants received feedback about their average accuracy, expressed as a percentage based on the mean response error (i.e., $100 \times(180$-mean error)/180).

On the $20 \%$ trials with attention ratings (modelled after Adam \& Vogel, 2017), memory test was followed by an attention probe, presenting the following question in white against a grey background: "How attentive were you on this trial?" atop of a white slider scale. The scale ranged from 1 to 9 , with $1=$ Not at all Attentive and $9=$ Very Attentive. Below the scale, a green integer digit (size $=40$ pixels) was shown as soon as participants moved the mouse on the scale and changed from one to nine according to the 
position of the mouse. Participants submitted their rating by clicking on the slider with the left mouse button. Then, there was a 1-s blank interval, followed by a $500 \mathrm{~ms}$ intertrial interval. Attention probes were balanced across the cue conditions (18 probes per condition and session) and pseudo-randomized to occur with two, three or four nonprobed trials in between. Because of a programming error, attention probes were distributed across the first 288 trials of a session, whereas no probes occurred in the last 72 trials $^{1}$.

Per session, there were four conditions: no-cue short, no-cue long, neutral-cue and retro-cue (100\% valid). Each condition had 90 trials per session, resulting in a total of 360 experimental trials. The conditions were randomly intermixed. Before the task, participants practiced for 20 trials (12 trials with the retro-cue and two trials per each other condition). Participants were instructed to remember color-location associations shown in the memory array. The instructions encouraged to heed the retro-cue and emphasized that the cue was $100 \%$ valid.

${ }^{1}$ This programming error was corrected in the next experiments. As the central results of Experiment 1 were replicated in Experiments 2-4, it is unlikely that the programming error had affected the results of Experiment 1 in any meaningful way. 


\section{Figure 2}

Conditions and Trial Timeline in Experiment 1.

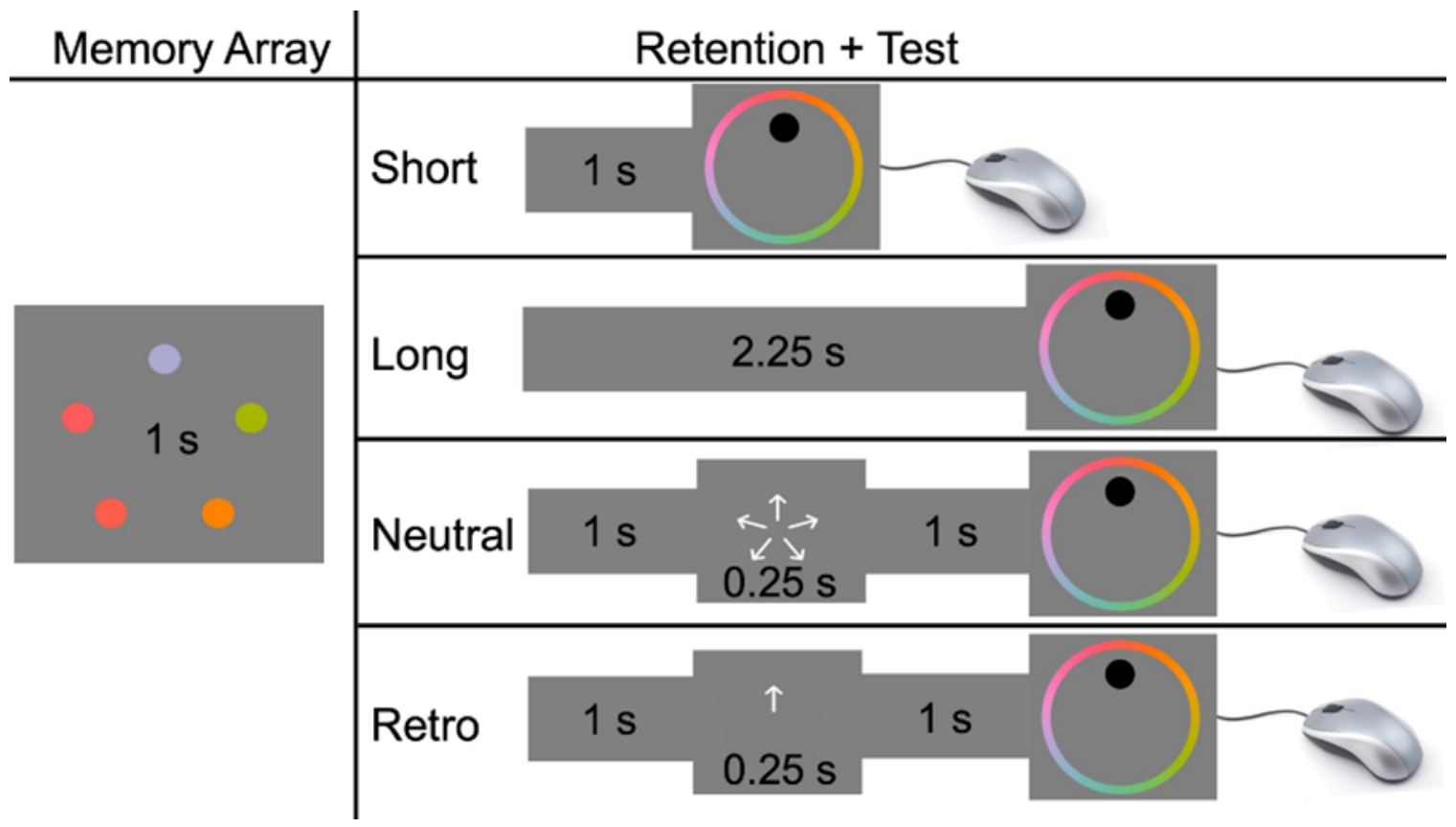

Note. Conditions were randomly intermixed during the experiment. The duration of each display is presented within the display in seconds. There is no time limit to the response in the test.

\section{Data Analysis}

The main dependent variables were (1) recall error, defined as the absolute distance between the reported color and the true color of the tested item, and (2) attention ratings ${ }^{2}$. We used Bayesian analyses of variance (BANOVA) and $t$-tests implemented in the BayesFactor package (R. D. Morey et al., 2018) with the default prior settings. We

${ }^{2}$ See Figure 7 for the distributions of attention ratings per condition levels across the experiments. 
regarded Bayes factors (BFs) between 3 and 10 as substantial, and larger than 10 as strong evidence in favor of $\mathrm{H}_{1}$. Likewise, BFs between $.30-.10$ were interpreted as substantial, and lower than .10 as strong evidence in favor of $\mathrm{H}_{0}$ (Wagenmakers et al., 2011). Ambiguous evidence for $\mathrm{H}_{0}$ was indicated by BFs in the range of .30 to 1 and for $\mathrm{H}_{1}$ in the range from $\mathrm{BF}=1$ to 3 .

We excluded one participant with recall error $M=88^{\circ}\left(S D=52^{\circ}\right)$, as average error around $90^{\circ}$ is indicative of random responding, thus leaving a final sample size of $N$ $=19$. As neither recall error nor attention ratings differed as a function of session (see Supplemental Materials on the OSF), the data were collapsed across sessions. Recall error did not differ between trials with vs. without attention probes, indicating that the subset of attention-probed trials is an adequate representation of the whole dataset (see Supplementary Materials on the OSF, https://osf.io/z2dpc/?view_only=bda94be6c4994dbfa736e6f57a464fa5).

\section{Results}

\section{Memory Accuracy}

First, we assessed whether the retro-cue increased memory accuracy compared to the baseline conditions, replicating the retro-cue effect. This analysis includes data from all trials (with and without attention probes). The data are visualized in Figure 3A. A oneway BANOVA having recall error as the dependent variable, subject as a random effect, and condition as a predictor showed strong evidence for a main effect of cue condition $\left(\mathrm{BF}=4.65 \times 10^{9}\right)$. Paired $t$-tests revealed that recall error was credibly smaller in the retro-cue compared to all other conditions (retro vs. short: $\mathrm{BF}=2.4 \times 10^{3}$; retro vs. long: $\mathrm{BF}=1.1 \times 10^{4}$; retro vs. neutral: $\left.\mathrm{BF}=1.6 \times 10^{3}\right)$, whereas baseline conditions did not 
credibly differ between each other (short vs. long: $\mathrm{BF}=0.46$, short vs. neutral: $\mathrm{BF}=$ 0.38, neutral vs. long: 0.24$)$.

\section{Attention Ratings}

Second, we analyzed whether self-rated attention varied as a function of condition (visualized in Figure 3B). A one-way BANOVA having attention ratings as the dependent variable, subject as random effect, and condition as a predictor yielded strong support for a main effect of condition $(\mathrm{BF}=373)$. Paired comparisons with $t$-tests indicated that ratings were higher in the retro-cue compared to all other conditions (neutral-cue condition, $\mathrm{BF}=56$; no-cue short, $\mathrm{BF}=42$; no-cue long, $\mathrm{BF}=6.80$ ). Arousal from cue sudden onset cannot explain this boost since ratings between the no-cue long and neutral-cue conditions were statistically indistinguishable $(\mathrm{BF}=0.26)$. Furthermore, attention ratings did not differ between the no-cue short and no-cue long baselines $(\mathrm{BF}=$ 0.26), indicating that sustained attention did not recede with longer VWM maintenance. In sum, self-rated attention was similar across the no-cue and neutral-cue conditions, but it was higher following a retro-cue. 


\section{Figure 3}

Results of Experiment 1 Showing Retro-Cue Effects on Recall Error (Panel A) and Attention Ratings (Panel B), and Similar Retro-Cue Benefits over all Levels of Self-Rated Attention (Panel C Presents Observed Data and Panel D Model's Predictions).

A

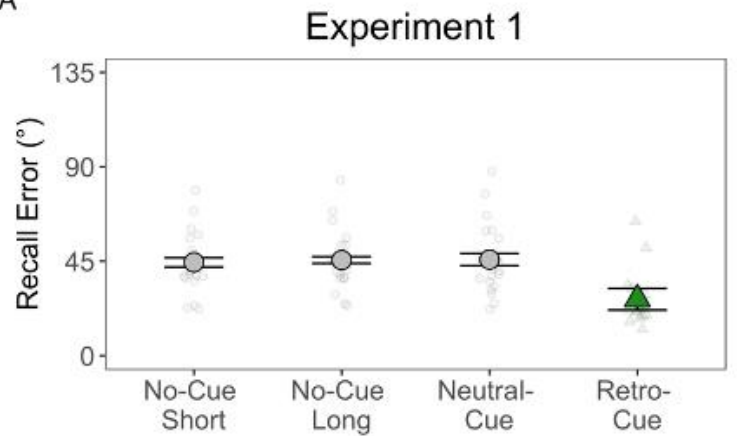

$\mathrm{B}$

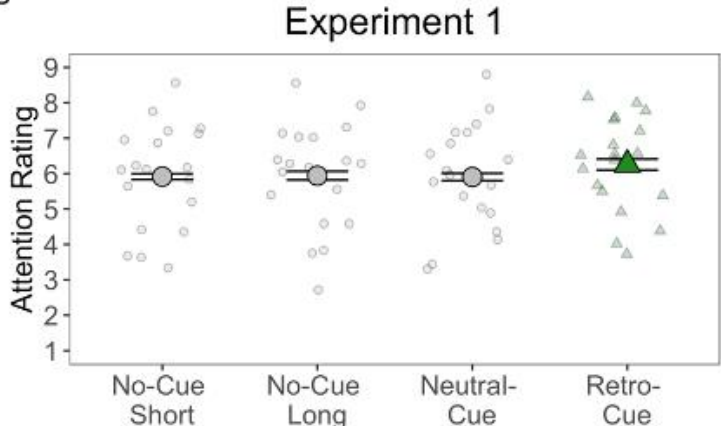

C

Experiment 1

Data

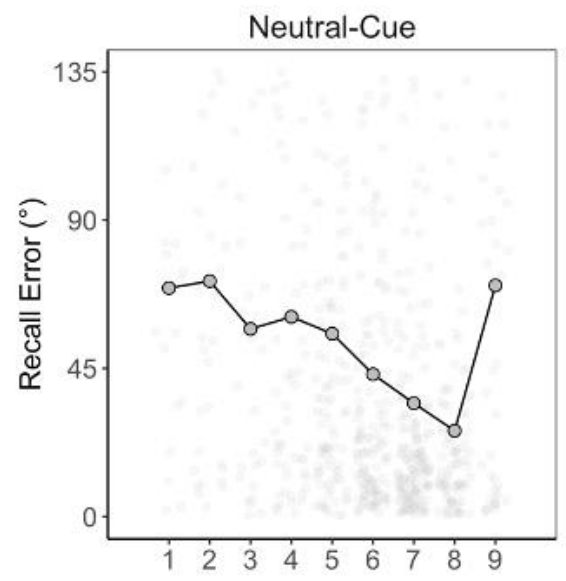

No-Cue-Merged

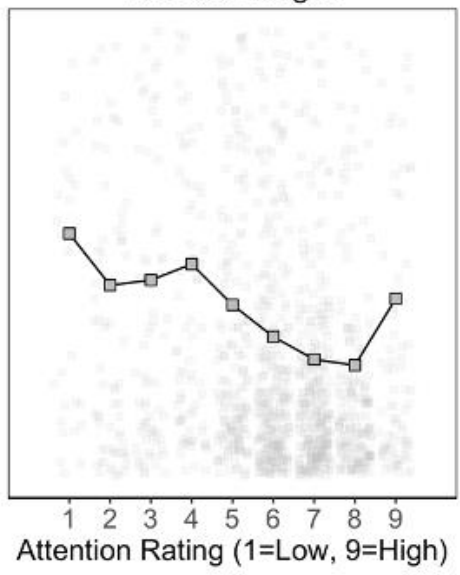

Retro-Cue

D

Experiment 1

Predictions
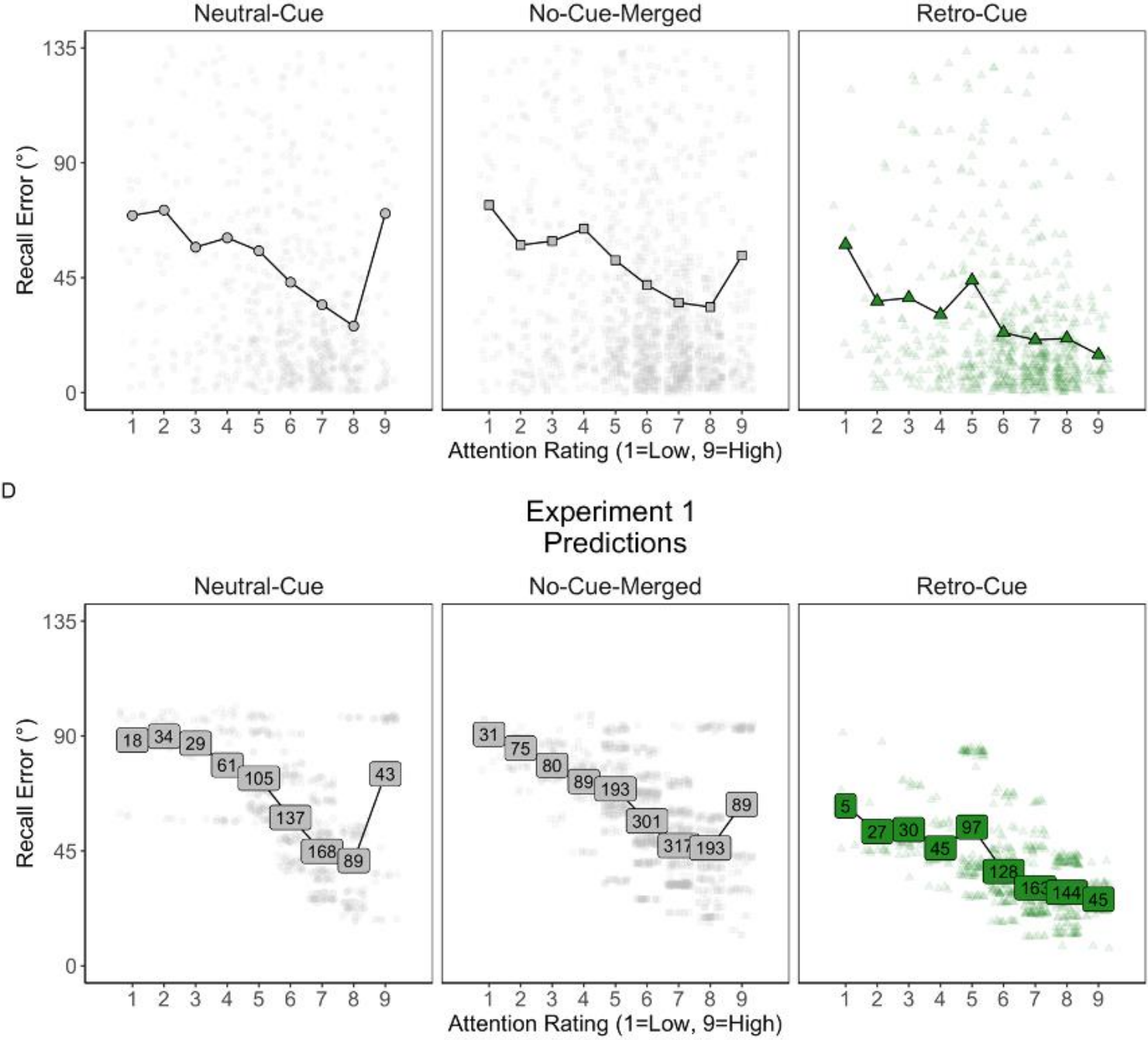
Note. The small dots in Panels A and B represent the individual's average in each condition. The large dots represent the condition mean. Note that in Panel A, the recall error data are aggregated across the attention probe condition (i.e., trials with and without the attention ratings). In Panels $\mathrm{C}$ and $\mathrm{D}$, the dot clouds are individual observations. NoCue-Merged condition represents the collapsed data of the No-Cue Short and No-Cue Long conditions. In Panel D, labels replace points at condition means and display the number of observations per attention rating level entered in the model (number of observations are identical to those displayed in Panel C).

\section{Joint Effects of Attention Rating and Cueing on Accuracy}

To answer our main research question, we modelled the joint effects of attention and cueing on task performance, using only the data from trials with attention probes. For this analysis we collapsed the data of the two no-cue conditions since they produced comparable results $(\mathrm{BF}=0.92)$. Figure $3 \mathrm{C}$ visualizes the relation of recall error and attention ratings for each cue condition: neutral-cue, merged no-cue, and retro-cue in the observed data and Figure 3D visualizes the model's predictions.

Our model, a hierarchical Bayesian regression model implemented in the brms package (Bürkner, 2018) in R (R Core Team, 2018), had cue condition (no-cue, neutralcue, and retro-cue) and mean-centered attention ratings as predictors, and the signed distance between the response and the true color in each trial as the dependent variable (hereafter response deviation). The distribution of response deviations can be described by a von Mises, which is the analogue of a normal distribution in circular space. The two parameters of von Mises distribution are the location parameter $\mu$ (corresponding in our model to the target color), and the concentration parameter $\kappa$ (corresponding in our model to the variability of the response deviations around the true target color). The $\kappa$ parameter describes the precision of the distribution: The higher the value of $\kappa$, the narrower the distribution becomes around $\mu$, whereas when $\kappa=0$, the distribution is completely flat 
and resembles a uniform distribution. In our model, we allowed our predictors to modulate the value of $\kappa$, setting $\mu=0$ because we centered all deviations on the target value. We used the package's default links for $\kappa(\log )$ and default priors for the von Mises distribution - normal $(5.0,0.8)$ for fixed effects and student_t $(3,0,10)$ for random effects, and we sampled four chains with 2000 iterations (1000 first iterations were discarded as warm-up). For the population-level (i.e., fixed) effects, smallest number of effective samples was 886 and $\hat{R}=1.00$, indicating good model convergence.

This analysis generates a distribution of the likely values of the parameters in the model, which are termed the posterior of the parameter. The summary statistics of the parameter posterior distributions are reported in Table 1 . We considered an effect as credible if the $95 \%$ Highest Density Interval (HDI) of the parameter's posterior did not include zero. The HDI represents the range of values that the parameter could reasonably assume to describe the data.

As shown in Table 1, there was a credible main effect of self-rated attention on memory precision $(\kappa)$, such that at higher attention ratings memory precision was higher. Compared to the merged no-cue baseline, precision was higher in the retro-cue condition, but not in neutral-cue trials. None of the interaction terms was credible, suggesting independent contributions of between-trial spontaneous attentional fluctuations, as reflected in attention reports, and selective focused attention, as reflected in the retro-cue benefits. 


\section{Table 1}

Posterior Estimates of the Regression Predictor Coefficients in Experiments 1, 2 and 4. Conditions and Coefficients with Credible Gains (Positive Sign) and Detriments (Negative Sign) on Recall are in Boldface.

\begin{tabular}{|c|c|c|c|c|c|c|}
\hline \multirow[t]{2}{*}{ Parameter } & \multicolumn{2}{|c|}{ Experiment 1} & \multicolumn{2}{|c|}{ Experiment 2} & \multicolumn{2}{|c|}{ Experiment 4} \\
\hline & Mean & $95 \% \mathrm{HDI}$ & Mean & $95 \% \mathrm{HDI}$ & Mean & $95 \% \mathrm{HDI}$ \\
\hline Intercept & 0.56 & {$[0.19,1.05]$} & 0.94 & {$[0.63,1.32]$} & 0.26 & {$[-0.08,0.42]$} \\
\hline Attention (+) & 0.85 & {$[0.57,1.14]$} & 0.56 & {$[0.32,0.81]$} & 0.49 & {$[0.36,0.63]$} \\
\hline Neutral Cue & -0.06 & {$[-0.35,0.22]$} & -- & -- & -- & -- \\
\hline Retro Cue (+) & 0.74 & {$[0.56,0.92]$} & $\mathbf{0 . 8 8}$ & {$[0.50,1.26]$} & 0.87 & {$[0.69,1.05]$} \\
\hline Rating Before (-) & -- & -- & -0.90 & {$[-1.12,-0.69]$} & -0.79 & {$[-0.99,-0.61]$} \\
\hline Attention $\times$ Neutral Cue & 0.24 & {$[-0.10,0.63]$} & -- & -- & -- & -- \\
\hline Attention $\times$ Retro Cue & 0.09 & {$[-0.28,0.48]$} & -0.06 & {$[-0.37,0.24]$} & -0.08 & {$[-0.25,0.10]$} \\
\hline Attention $\times$ Rating Before & -- & -- & -0.16 & {$[-0.41,0.10]$} & 0.06 & {$[-0.15,0.27]$} \\
\hline Retro Cue $\times$ Rating Before $(+)$ & -- & -- & 0.72 & {$[0.37,1.07]$} & 0.61 & {$[0.39,0.84]$} \\
\hline Attention $\times$ Retro Cue $\times$ Rating Before & -- & -- & 0.29 & {$[-0.17,0.74]$} & 0.21 & {$[-0.05,0.46]$} \\
\hline
\end{tabular}

Note. (+) denotes performance gains, and (-) denotes performance detriments. The regression weights reflect the change in the concentration parameter $\kappa$ of the von Mises distribution. Larger values of $\kappa$ indicate a narrower distribution and, accordingly, higher recall precision. The values are on the logarithmic scale. Square brackets contain the lower and upper Highest Density Interval (HDI) of the posterior (credible effects do not include the zero). "Rating Before" refers to ratings placed prior to memory test. All models included the full attention rating scale (1-9). Outlier participants were excluded from the models of Experiments $1(n=1)$ and $4(n=$ 5). 


\section{Discussion}

Experiment 1 demonstrated that retro-cueing increases both memory accuracy and subjective experience of attention. Modelling the effect of cueing and attention on performance showed that both these predictors affected memory accuracy separately. In other words, cue-enabled selection contributed to a steady increment in accuracy across the whole range of self-rated attentional levels. The lack of interaction between subjective sustained attention level and selective focused attention via retro-cueing indicates that the ability to use the retro-cue does not depend on one's attentional state.

The main effect of retro-cueing on self-rated attention indicates that participants experience higher attentiveness after being able to deploy focused attention. The contributions of other sources towards changes in self-rated attention with planned comparisons showed that, firstly, the increase in subjective attention was not due to the presentation of a sudden-onset stimulus which could drive arousal, as attention reports did not increase after neutral cues. Secondly, comparisons of the no-cue short (1-s retention) and no-cue long (2.25-s retention) baselines revealed no decline in ratings with longer maintenance. Thus, retro-cueing contributed to an increase in self-rated attention above its baseline level.

However, because attention ratings were provided after placing the memory responses, participants may have incorporated the evaluation of their performance into their attentional reports. In other words, because retro-cues were helpful for memory accuracy, participants may have perceived that their performance was better and included this experience in their attentional ratings. To assess whether participants' attention ratings are based on perceived attentional state or are biased by the evaluation of their 
recall performance, Experiment 2 solicited attention reports either before or after the memory test.

\section{Experiment 2}

In Experiment 2, we assessed whether the introspection on subjective attention incorporated participants' evaluation of their behavioral performance. For this purpose, we again collected attention ratings on retro- and no-cue trials, additionally splitting the rating time-points between before and after the memory test. We hypothesized that ratings placed before the memory test could only be influenced by the participants' attentional state, whereas ratings collected after the memory test could be influenced by attentional state and/or behavioral evaluation. If behavioral evaluation was the driving variable producing increase in attentional ratings after retro-cues compared to the other conditions, then we should observe this effect after the memory test, but not before it.

\section{Participants, Materials, and Procedure}

A new sample of twenty university students from the Zurich area $\left(M_{\text {Age }}=22.6\right.$, $S D=3.74,16$ women) completed two sessions for a compensation of $30 \mathrm{CHF}$ or course credit. The experiment was identical to Experiment 1, except for the following modifications. First, the experimental conditions were reduced from four to two: Retrocue vs. the no-cue short baseline (50\% each). Out of 360 trials per session, we administered attention probes on 72 trials, resulting in 18 probed trials per each design cell (i.e., Cue $\times$ Probe Time-Point) in each session, amounting to a probe rate of $20 \%$. The following timing variables were changed: The inter-trial interval was increased to $1500 \mathrm{~ms}$, and waiting time after the placement of attention ratings was set to 0 . Both these changes aimed to reduce the impact of the attention ratings on performance, given 
that providing attention ratings before the memory test may unsettle participants' orientation in the experiment flow. To minimize visual load, attention probes solely presented the rating scale with the numerical anchors and their annotation $1=$ Not at all Attentive, and on the right, at $9=$ Very Attentive. Participants practiced for 30 trials. They completed a total of 720 trials across the two sessions.

\section{Results}

For the analyses, the data were collapsed across sessions, as neither rated attention nor recall accuracy differed as a function of session (see Supplementary Materials on the OSF).

\section{Memory Accuracy}

First, we analyzed whether retro-cueing increased recall accuracy considering three sets of data: non-probed trials, trials in which attention was rated before the memory test, and trials in which ratings were entered after the test. The data are visualized in Figure 4A. A Cue (No-Cue vs. Retro-Cue) $\times$ Rating Time-Point (No Rating, Rating Before, or Rating After) BANOVA having recall error as dependent variable and subject as a random effect yielded largest support for a model with the main effects of Cue and Rating Time-point and their interaction (see Table 2 for BFs and ratios of evidence for each predictor). With retro-cues, there was no credible difference in performance when attention probes appeared after the test $v s$. probe-absent trials $(\mathrm{BF}=$ 0.23 ), and there was ambiguous evidence for worse performance when attention probes were presented before the test $v s$. probe-absent trials $(\mathrm{BF}=2.52)$, as well as when attention probes appeared before $v s$. after the memory test $(\mathrm{BF}=1.44)$. In contrast, in nocue trials, there was strong evidence that placing attention reports negatively affected 
performance when the probes occurred before the memory test compared to probe-absent trials $\left(\mathrm{BF}=1.4 \times 10^{4}\right)$, and to trials in which attention reports occurred after the memory test $\left(\mathrm{BF}=5.1 \times 10^{4}\right)$. Recall did not differ in probe-absent trials $v s$. after the memory test $(\mathrm{BF}=0.24)$. Together, these results indicate that placing attention ratings after experiencing retro-cues did not credibly affect recall accuracy, whereas it was detrimental in no-cue trials. This is in line with previous studies demonstrating that retro-cues protect memory representations from performance decrements induced by a secondary task (Hollingworth \& Maxcey-Richard, 2013; Makovski \& Jiang, 2007; Makovski \& Pertzov, 2015; Matsukura et al., 2007; Rerko et al., 2014; Sligte et al., 2008; van Moorselaar,

Gunseli, et al., 2015).

\section{Table 2}

Results of the BANOVA on the Recall Error and Attention Rating in Experiment 2 Showing Credible Effects of All Predictors in the Recall Error but Only of Cue for Attention Ratings.

\begin{tabular}{|c|c|c|c|c|}
\hline \multirow[t]{2}{*}{ Model } & \multicolumn{2}{|c|}{ Recall Error } & \multicolumn{2}{|c|}{ Attention Ratings } \\
\hline & Model & Predictor & Model & Predictor \\
\hline Cue & $4.86 \times 10^{17}$ & $1.26 \times 10^{21}$ & $1.10 \times 10^{5}$ & $1.3 \times 10^{5}$ \\
\hline Rating Time & 14 & 38104 & 0.33 & 0.42 \\
\hline Cue + Rating Time & $1.85 \times 10^{22}$ & --- & $4.4 \times 10^{4}$ & --- \\
\hline Cue + Rating Time + Cue $\times$ Rating Time & $1.42 \times 10^{25}$ & 771 & $5.7 \times 10^{4}$ & 1.21 \\
\hline
\end{tabular}

Note. The Model-column presents the evidence (BF) for the specified model against the Null. The Predictor-column presents the evidence for the inclusion of the factor printed in italics when contrasted to the two main effects' model (credible effects, i.e., BF > 3, are printed in bold). 


\section{Figure 4}

Experiment 2 Results Showing Retro-Cue Effects on Recall Error (Panel A) and Attention Ratings (Panel B), as Well as a Constant Retro-Cue Benefit over All Levels of Self-Rated Attention (Panel C Presents the Observed Data and Panel D Model's Predictions)

A

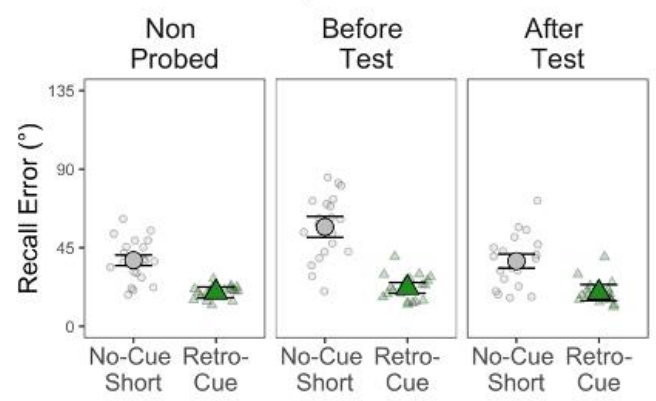

B

Experiment 2

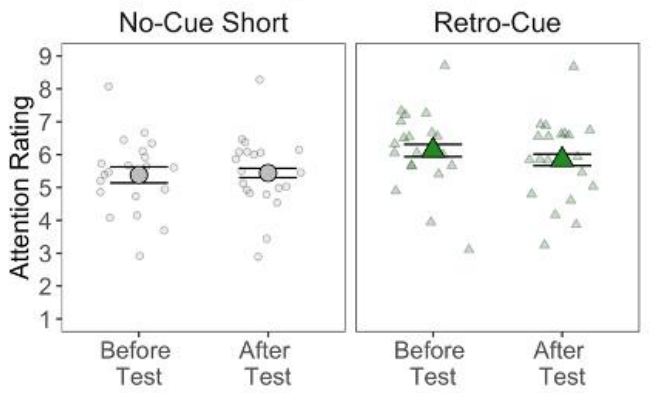

。

Experiment 2

Data
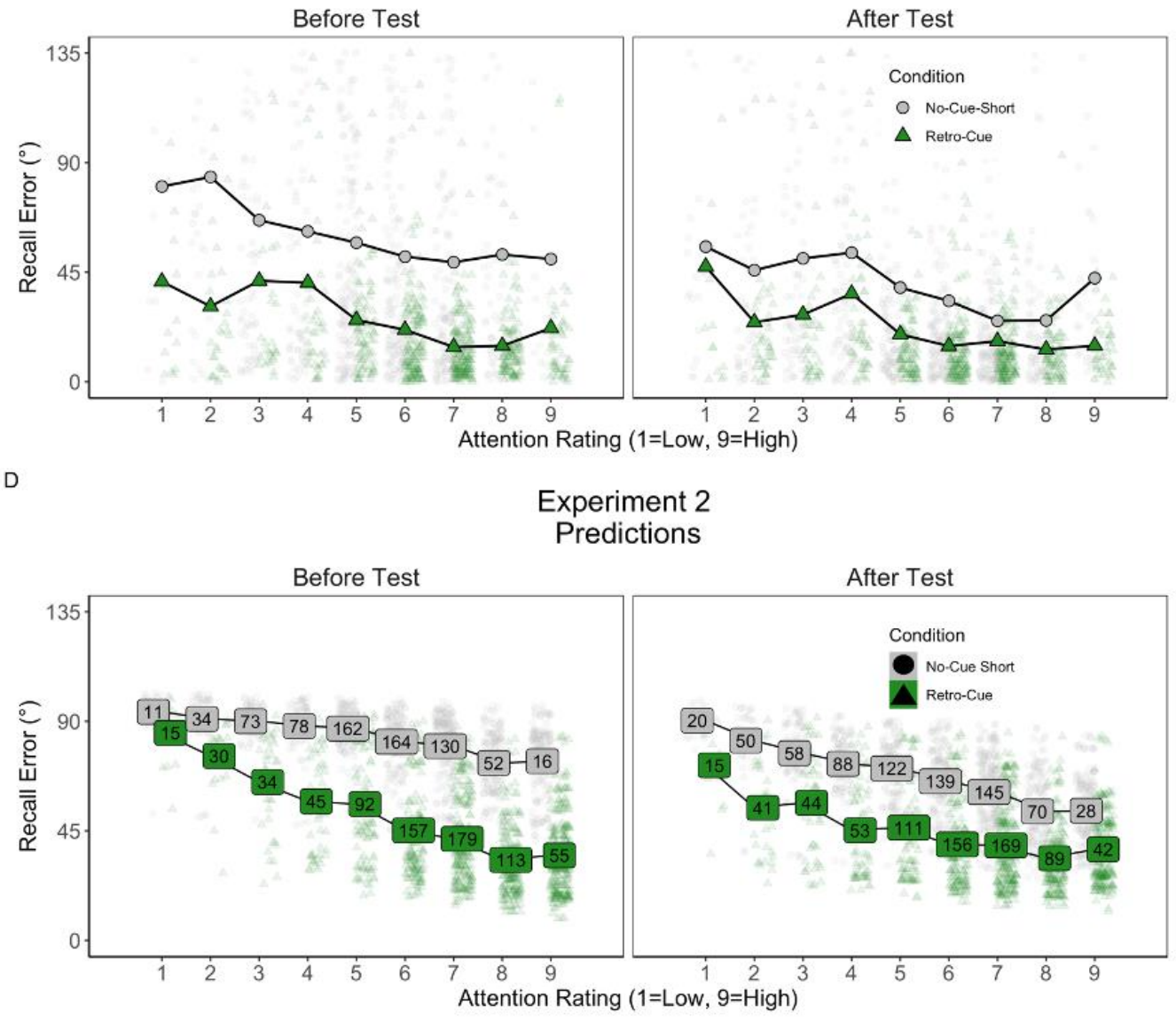

Note. Small dots in Panels A and B are each individual's average in that condition, and the large dots are the sample mean. Error bars are the $95 \%$ within-subjects' confidence intervals. In Panels $C$ and D, the dot clouds are the individual observations. Panel D displays the number of observations entered in the model. 


\section{Attention Ratings}

Further, we analyzed whether attention ratings differed as a function of cue condition and rating time-point ${ }^{3}$. These data are visualized in Figure 4B. Attention ratings were analyzed with a Cue $($ No-Cue vs. Retro-Cue) $\times$ Rating Time-Point (Rating Before vs. Rating After) BANOVA (with subject as a random effect). The model including only the main effect of cue was the best, indicating that retro-cueing increased participants' attention irrespective of the time-point of the rating (see Table 2). As there was ambiguous evidence for including the interaction term on the model, we conducted separate $t$-tests for each rating time-point. Attention was higher in the retro-cue compared to no-cue condition when ratings were placed before the test $(\mathrm{BF}=114)$, and after the memory test $(\mathrm{BF}=19)$. Thus, the ambiguous interaction stems from self-reported attention being somewhat higher when ratings were placed before the memory test. This goes against the notion that performance evaluation was driving the difference in ratings between the retro-cue and no-cue conditions.

\section{Joint Effects of Attention Rating and Cueing on Accuracy}

Lastly, we modelled the effects of cueing, rated attention levels, and rating timepoint on response deviation using the same approach and settings as in Experiment 1. Figure $4 \mathrm{C}$ visualizes the observed data as a relation of cue condition and attention ratings per rating time-point, and Figure 4D the model's predictions. The model showed an

\footnotetext{
${ }^{3}$ Ratings' distributions across experiments are visualized in Figure 7.
} 
adequate fit (for population-level (i.e., fixed) effects, $\hat{R}=1.00$ and effective samples > 932). Posterior distributions are summarized in Table 1.

There were credible main effects of attention rating, retro-cue and rating timepoint. The main effects of attention rating and retro-cue replicate the findings that memory precision was higher when attention was rated higher, as well as after retrocueing. Conversely, placing attention ratings before the memory test was associated with impaired performance. However, retro-cueing mitigated this deleterious effect of distraction before the memory test, as indicated by the credible interaction of retro-cue and the rating-before time-point.

Lastly, replicating Experiment 1, there was no credible interaction between retrocue and attention. Thus, we again observed constant retro-cue benefits across all levels of self-rated attention, which argues for independent contributions of overall sustained attention level and selective focusing to VWM performance.

\section{Discussion}

In Experiment 2, we aimed to rule out that participants' attention appraisals were biased by performance evaluation. If performance evaluation was the driving variable for the increase in attention ratings following retro-cues, then the difference between no-cue vs. retro-cue conditions should only emerge when ratings were submitted after the test, but not before it. The results refuted the idea that self-rated attention was driven by performance evaluation, as the increase in ratings was credible also in the condition where these were placed prior to submitting the memory response. 
Critically, we again observed that retro-cue benefits were of similar magnitude at all levels of self-rated attention. This finding, similar to Experiment 1, indicates that selective and sustained attention produced independent effects on VWM performance.

\section{Experiment 3}

Experiment 2 demonstrated that attention ratings plausibly reflect introspection on one's attention, instead of performance evaluation, as ratings placed before and after memory testing were similarly affected by retro-cueing. In Experiment 3, we further probed the role of performance evaluation in attentional introspection by using invalid retro-cues. Experiment 3 was carried out online. One group of participants was asked to place all their ratings prior to memory testing $(n=105)$, whereas the other group placed all ratings after memory testing $(\mathrm{n}=122)$. This allowed us to replicate the results of Experiment 1 and 2. The crucial manipulation consisted in the cue validity: One-third of the cues were now invalid (i.e., $33 \%$ invalid cues compared to $66 \%$ valid cues). When participants are encouraged to focus attention on one memory representation, but are then tested on one of the non-cued items, this produces a performance cost (Astle et al., 2012; Gözenman et al., 2014; Gressmann \& Janczyk, 2016; Griffin \& Nobre, 2003; Gunseli et al., 2015; Pertzov et al., 2013; Souza, Thalmann, et al., 2018; van Moorselaar, Olivers, et al., 2015, 2015), which has been interpreted as evidence that non-cued items are removed from VWM thereby freeing capacity (Gunseli et al., 2018; Souza, Rerko, \& Oberauer, 2014; Williams et al., 2013). Accordingly, if participants substantially drew on their performance when appraising their attention, that should result in ratings in the invalidcue condition (that produces costs to response accuracy) being lower compared to the nocue trials and the valid retro-cue trials, which produce better memory performance. Note 
that only when ratings are placed after the memory test, the effect of invalid cues becomes evident, as prior to the memory test, participants do not know whether the retrocue is valid or invalid.

\section{Participants}

Participants were English speakers, resident in the UK, U.S., or Australia $(N=$ $227, M_{\text {Age }}=26.62, S D=5.38 ; 116$ women, 110 men, and 1 other) recruited for an online study via the Prolific platform. The study lasted 30 min and participants were compensated with 4.5 British Pounds. We started by collecting data of 74 participants, and we increased sample size in two additional waves $(n=57, n=96)$ aiming to obtain credible evidence for or against an effect of cue validity on attention ratings (i.e., BF > 3). This tiered data-collection approach is feasible when Bayesian estimation is used (Rouder, 2014). Unfortunately, even after collecting this larger sample, results were still ambiguous for some of the condition contrasts.

\section{Procedure}

The experiment was programmed in lab.js (Henninger et al., 2020) which is a HTML and JAVASCRIPT based language for programming online psychological experiments. As in the previous experiments, participants memorized the color-location associations of five colored discs. Before the memory array, a white fixation cross was shown (font-size $50 \mathrm{pts}$ ) for $500 \mathrm{~ms}$. Next, the five colored discs (radius $=25$ pixels), arranged equidistantly around an invisible circle (radius 150 pixels), were displayed for $500 \mathrm{~ms}$. In the no-cue condition, the memory test followed after a 1-s retention interval. In the memory test, a color wheel (randomly rotated on each trial) and the probe item (a dark-grey dot appearing in one of the memory locations) appeared together with the 
mouse cursor. In the retro-cue condition, the 1-s retention interval was followed by an arrow cue, displayed for $250 \mathrm{~ms}$, pointing to the location of the to-be-tested item, which was then followed by another 1-s post-cue interval before the onset of the memory test. The cue was valid on two thirds of the trials (i.e., it correctly indicated the tested location), whereas in one third of the trials, the cue pointed to a non-tested item. We selected a $66.6 \%$ validity as previous research from our lab observed both valid-cue benefits and invalid-cue costs using this proportion (Souza, Thalmann, et al., 2018) and we wanted to maximize the number of invalid trials without compromising the observation of retro-cue benefits. Participants completed 200 randomly intermixed types of trials: 80 no-cue trials, 80 valid retro-cue trials, and 40 invalid retro-cue trials.

Attention ratings were solicited on $20 \%$ of the trials (40 probes): 16 probes following no-cue trials, 16 probes in valid trials, and 8 probes in invalid trials. In the instruction, participants were informed that the cue is often, but not always valid. As in previous experiments, the dependent variables were recall error and attention ratings. The independent variable Cue (No-Cue, Invalid, Valid) was manipulated within subjects, and the dependent variable Rating Time-Point (Before vs. After Test) was manipulated between subjects. Participants were randomly assigned to the two groups. There were $n=$ 105 participants in the Rating-Before group and $n=122$ in the Rating-After group. Preliminary inspection of the data revealed that $n=11$ participants had a mean recall error $>80$ and $n=19$ participants only reported one attention rating value throughout the experiment (rating $S D=0$ ); two participants met both criteria. As mean recall error close to $90^{\circ}$ indicates that participants are guessing, and data with zero variance likely represents invalid survey response behavior (Curran, 2016), we conducted 
analyses both on the whole dataset and on a subset without these outlier participants. Unless otherwise indicated, the pattern of the results did not differ. Prior to the main analyses, we evaluated whether recall error differed on trials with vs. trials without attention probes. The results showed that probe presence produced larger recall errors in the Rating-Before test group, compared to the trials that did not solicit attention ratings, but this effect did not occur in the Rating-After test group, replicating the results of Experiments 1 and 2. These analyses are reported in Supplemental Materials on the OSF.

\section{Results}

\section{Memory Accuracy}

Recall error for the subset of attention-probed trials is visualized in Figure 5A. Recall error in these trials was assessed with a Cue (No-Cue, Invalid, or Valid) $\times$ Rating Time-Point (Before vs. After) BANOVA. The best model included only the main effect of cueing (see Table 3). Comparisons of models' BF ratios showed strong evidence for the role of cueing, whereas there was evidence against the role of rating time-point and ambiguous evidence against their interaction. In summary, invalid retro-cues increased recall error and valid retro-cueing reduced recall error compared to the no-cue condition but recall error did not credibly differ between the groups of participants that rated their attention before vs. after the memory test. Repeating the analysis excluding the subset of outlier individuals showed similar results: ambiguous evidence for an interaction $(\mathrm{BF}=$ 2.16) and rating time-point $(\mathrm{BF}=0.66)$, but the evidence for the role of cueing remained very strong $\left(\mathrm{BF}=6.65 \times 10^{83}\right)$. 


\section{Figure 5}

Results of Experiment 3 Showing Cueing Benefits and Costs on Recall Error (Panel A) but Mostly Cue Benefits on Attention Ratings (Panel B).

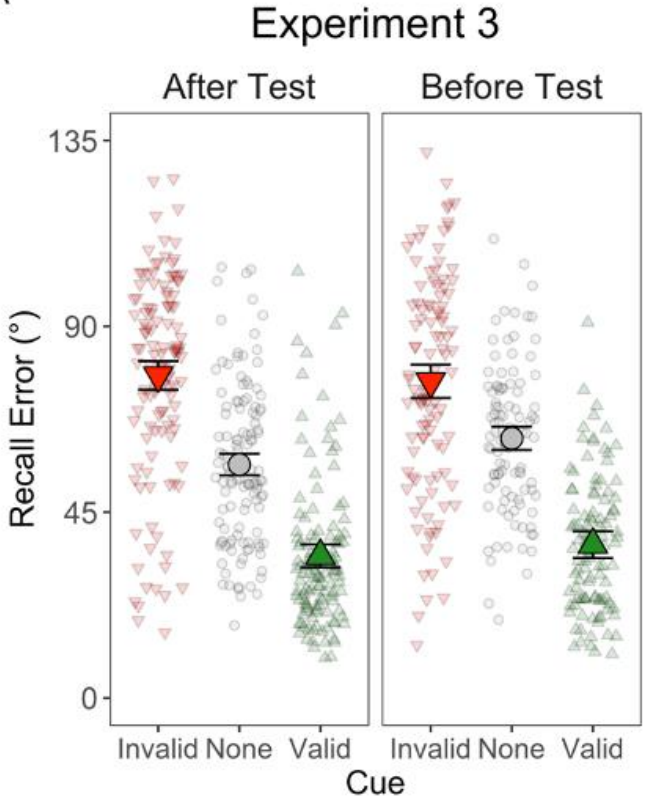

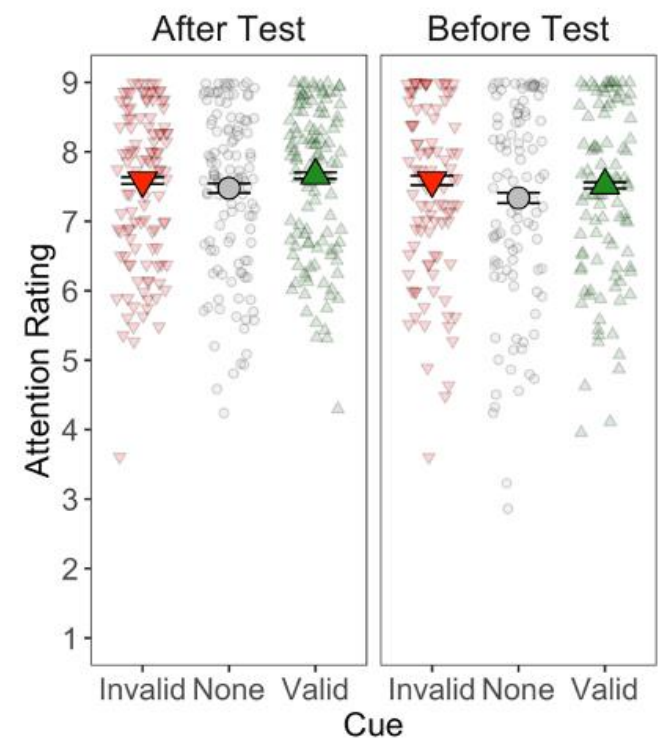

Note. Small dots in Panels A and B (full sample, $N=227$ ) represent each individual's average in that condition. The large dots represent the sample mean. Error bars are the 95\% within-subjects' confidence intervals.

\section{Table 3}

Results of the BANOVAs on the Recall Error and Attention Ratings in Experiment 3 Showing Only a Credible Effect of Cue both on Recall Error and Attention Ratings.

\begin{tabular}{|c|c|c|c|c|}
\hline \multirow[t]{2}{*}{ Model } & \multicolumn{2}{|c|}{ Recall Error } & \multicolumn{2}{|c|}{ Attention Ratings } \\
\hline & Model & Predictor & Model & Predictor \\
\hline Cue & $3 \times 10^{89}$ & $4.86 \times 10^{89}$ & $4 \times 10^{7}$ & $3.66 \times 10^{7}$ \\
\hline Rating Time & 0.17 & 0.27 & 0.58 & 0.47 \\
\hline Cue + Rating Time & $8.46 \times 10^{88}$ & --- & $1.89 \times 10^{7}$ & --- \\
\hline Cue + Rating Time + Cue $\times$ Rating Time & $3.77 \times 10^{88}$ & 0.44 & $2.16 \times 10^{7}$ & 1.13 \\
\hline
\end{tabular}

Note. The Model-column presents the evidence (BF) for the specified model against the Null. The Predictor-column presents the evidence for the inclusion of the factor printed in italics when contrasted to the two main effects' model (credible effects, i.e., BF > 3, are printed in bold). The models used the full dataset $(N=277)$. 


\section{Figure 6}

Results of Experiment 3. For the After-Test Group, Retro-Cue Benefits were Constant over Self-Rated Attention Levels. However, for the Before-Test Group, Retro-Cue Benefits Increased with Self-Rated Attention.

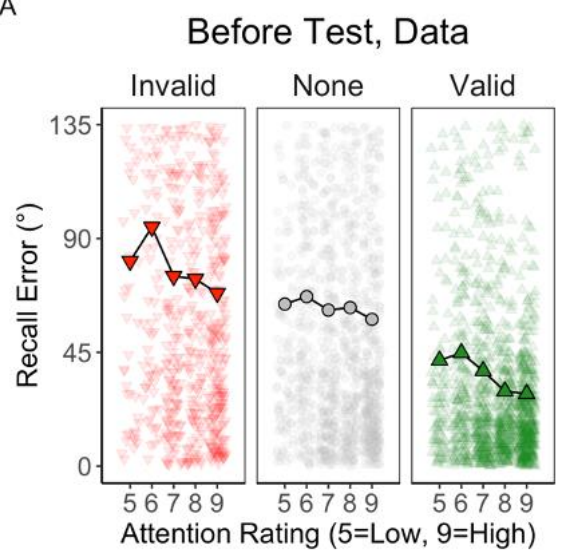
After Test, Data

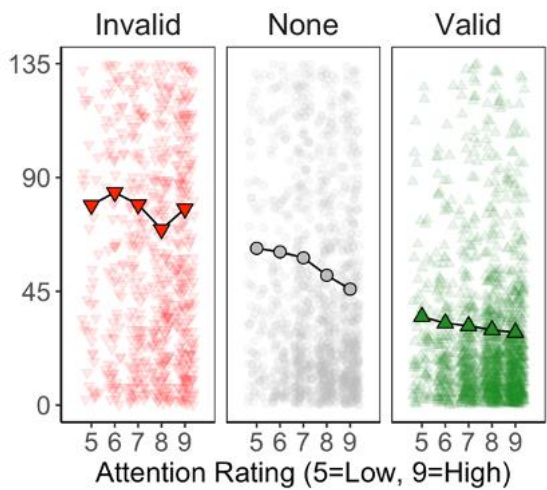

C

Before Test, Predictions

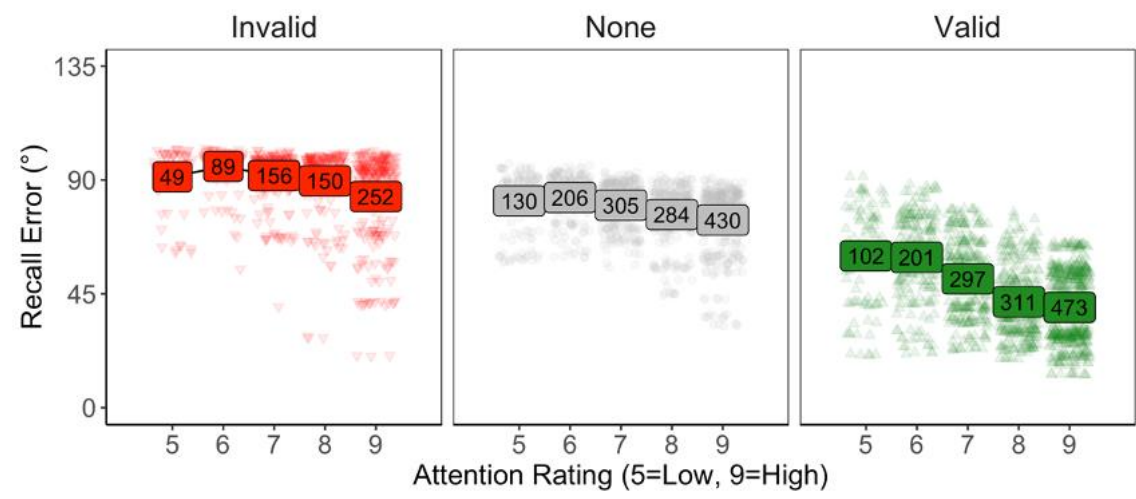

D

After Test, Predictions

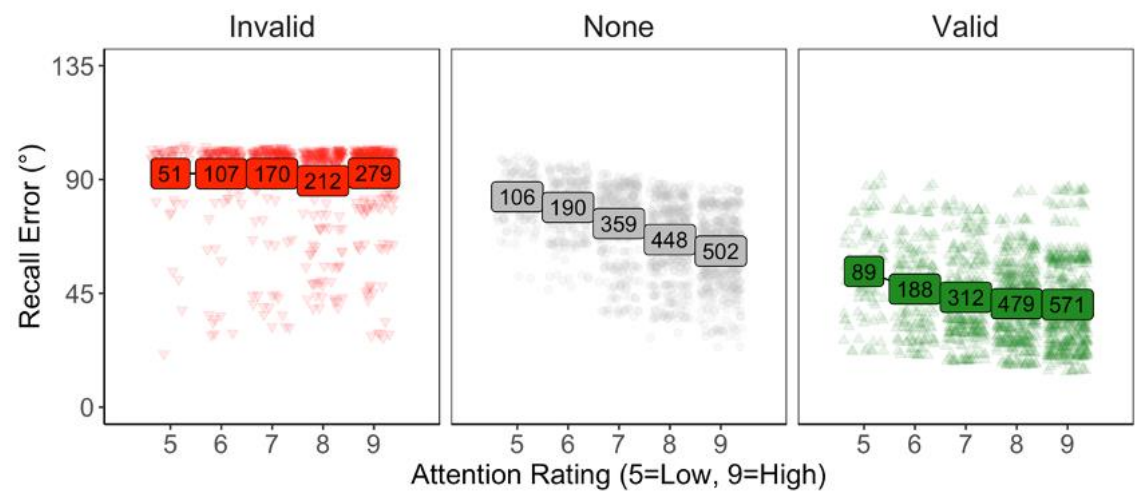

Note. The dot clouds are single observations/predictions; these data are from the subsample $(N=199)$ without outlier participants, as used in the reported models. Panels $\mathrm{C}$ and $\mathrm{D}$ display the number of observations per each condition combination level, the labels occupy predictions' mean values' place. 


\section{Attention Ratings}

Figure 7 presents the distribution of attention ratings in each condition. As can be seen in this figure, participants mostly used the upper range of the rating scale (unlike in the previous experiments, for which a normal distribution was observed). This may be due to the data being collected online, and participants being cautious to report inattentiveness, particularly given that in Prolific payment in several studies is contingent on participants passing attention checks.

Mean attention ratings as a function of cue condition and rating time-point are visualized in Figure 5B. To gauge the influence of cueing on self-rated attention, we ran a BANOVA with Cue (No-Cue, Invalid, Valid) $\times$ Rating Time-Point (Before vs. After). The best model was the one with only a main effect of cueing (see Table 3). Comparison of models' ratios showed strong evidence for the effect of cueing, and ambiguous evidence against the effect of rating time-point and for inclusion of their interaction. To explore the source of the ambiguous interaction, we conducted separate analyses for each rating time-point. Ratings placed before the memory test were aggregated to two levels (no cueing and retro-cueing), reflecting the notion that before memory testing, cue validity is unknown to the participant. A $t$-test supported a difference between these conditions: Ratings after retro-cueing were credibly higher $(M=7.54, S D=1.3)$ than in no-cue trials $(M=7.33, S D=1.5), \mathrm{BF}=1.4 \times 10^{3}$. Thus, cue presence (without knowledge of its validity) again increased participants' attention. For the subset of post-test ratings, a one-way BANOVA having subject as random effect revealed substantial evidence for the effect of cueing $(\mathrm{BF}=449)$. Here, mean ratings were lowest in no-cue trials $(M=7.47$, 
$S D=1.21$ ), and higher in retro-cue trials (Invalid-Cue: $M=7.58, S D=1.14$; Valid-Cue: $M=7.65, S D=1.11)$. A $t$-test confirmed that ratings credibly differed between No-Cue and Valid-Cue $(\mathrm{BF}=282)$ conditions. However, there was ambiguous evidence for a difference between the No-Cue and Invalid-Cue $(\mathrm{BF}=1.80)$ conditions and ambiguous evidence for a difference in ratings between Valid- and Invalid-Cue conditions, which nearly reached credibility $(\mathrm{BF}=2.43)$.

\section{Figure 7}

Proportion of Observations per Attention Rating Level and Experimental Condition in Experiments 1-4 Showing More Bell-Shaped Distributions in Experiments 1, 2, and to some Extent Experiment 4, than in Experiment 3.

A

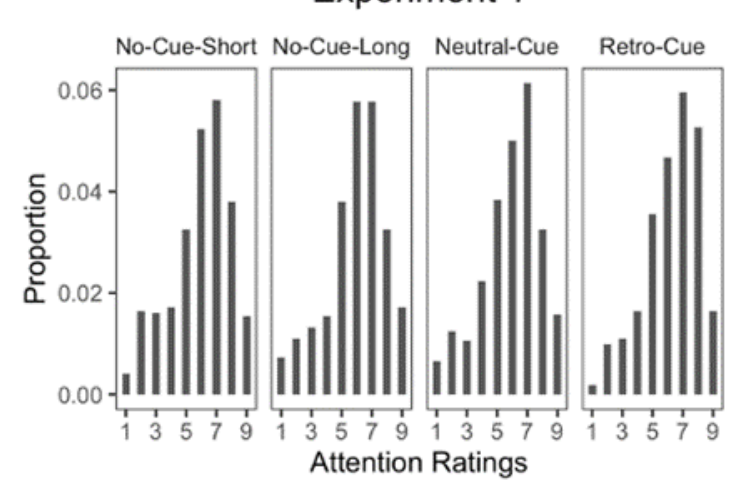

C

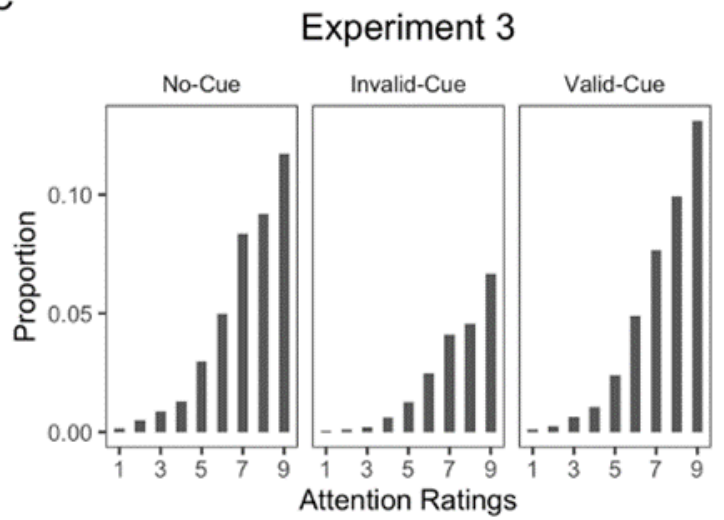

B

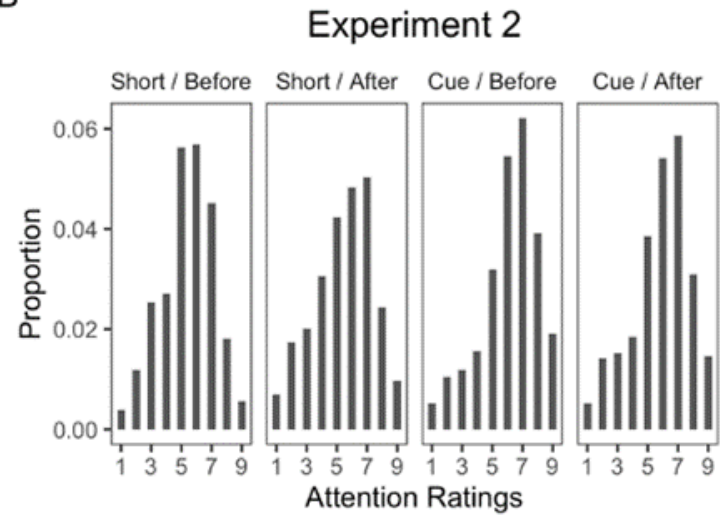

D

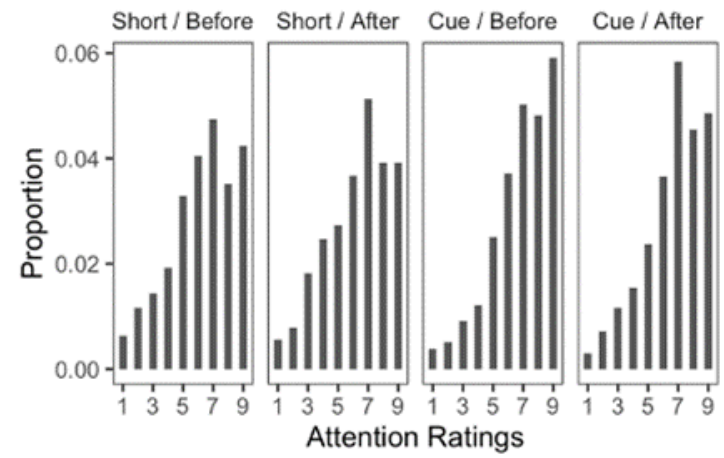

Note. Panels A, C and D visualize ratings' distributions for the samples without outlier participants (A: $N=19, \mathrm{C}: N=199, \mathrm{D}: N=93$ ). 
Together, these results indicate that attention ratings are higher following the retro-cue than in the no-cue condition, replicating our previous experiments. Attention reports after invalid cues were somewhat higher than in no-cue trials, but not credibly so, even after we collected over 100 participants in the condition. So far, these results suggest that behavioral evaluation may play some role in attention ratings: Ratings were somewhat lower if the cues were invalid compared to valid. However, the performance evaluation hypothesis predicts that they should actually be lower in the invalid than in the no-cue condition, which they were definitely not (i.e., a one-tailed $t$-test provided evidence against invalid-cue ratings being lower than in the no-cue condition, $\mathrm{BF}=$ 0.012). Another critical piece of evidence for testing this hypothesis is provided by assessing the correlation between attention ratings and performance, which will be reported next.

\section{Joint Effects of Attention Rating and Cueing on Accuracy}

As in previous analyses, we modelled the response deviations in a Bayesian hierarchical regression having Cue (No-Cue, Invalid, Valid), Rating Time-Point (Before vs. After), and mean-centered self-rated attention level as predictors. Figure 6 visualizes the individual observations and model's predictions separately for the Before- $(6 \mathrm{~A}, \mathrm{C})$ and After-Test (6B, D) attention rating time-points. As attention ratings below five were very scarce (see Figure 7), we conducted the regressions on the data of trials in which ratings were 5 and higher.

We report the analysis (see Table 4) without the outlier participants (i.e., mean recall error $>80$ and attention rating $S D=0, n=28$; analysis sample $N=199$ ) because individuals with low variance in the self-rated attention or recall error could bias it. 
Results of the model with the full sample are presented in the Supplementary Materials available on the OSF ${ }^{4}$. The regression model (population-level effects: $\widehat{R}=1.00$, effective samples > 1819) showed that, replicating Experiments 1 and 2, high attention ratings and valid cueing improved memory precision. Invalid-cueing produced costs in line with the hypothesis that participants dropped the non-cued items from VWM (Souza, Rerko, \& Oberauer, 2014). Likewise, submitting attention ratings before the memory test impaired memory precision. There was a credible two-way interaction between Attention and Valid-Cue, and a credible three-way interaction between Attention, Valid-Cue, and Before-Test Rating Time-Point. To disentangle the three-way interaction, we ran separate regressions per rating time-point (population-level effects; Before: $\widehat{R}=1.00$, effective samples > 1547, After: $\hat{R}=1.00$, effective samples $>1581$, see Table 4). For both rating time-points, high attention ratings and valid retro-cues increased precision, whereas invalid cues reduced it. Clarifying the source of the three-way interaction in the full model, there was a credible interaction of Attention $\times$ Valid-Cue when ratings were placed before the memory test. Thus, contrary to Experiment 2 (in the condition where ratings were also collected before the test), higher attention ratings went along with a

\footnotetext{
${ }^{4}$ The results' pattern was similar in the sense that a credible three-way interaction was observed. However, this interaction could not be parsed, as separate analyses for RatingBefore and Rating-After time-points did not reveal credible interaction terms. This could be attributed to respondents with low variance in the self-rated attention or recall error biasing the analysis.
} 
larger cue benefit. This result is puzzling because it does not replicate across Experiments 1, 2, and for the after-test group in Experiment 3.

One of the main aims of Experiment 3 was to test whether an interaction emerged between Attention and Invalid-Cue when ratings were collected post-test: If participants incorporated their performance evaluation into their attention reports, then the relationship between rated attention and performance should increase. To address this prediction, we conducted a directional test whether the covariation of participants' attention ratings increased at the after- compared to before-test rating placement, irrespective of cueing condition. Without specifying the cue condition, we computed a full model (main effects of Rating Time-Point, Attention, and their interaction) and a model with the main effects of these predictors only. As previously, we modeled the triallevel data and predictor Attention was mean-centered. To obtain a Bayes Factor from the models' comparison, the models had 50000 iterations (warmup =1000), and there were three chains. Comparison of the full model $\left(\hat{R}=1.00\right.$, effective samples $\left.>6.93 \times 10^{4}\right)$ with the two main-effects' model $\left(\hat{R}=1.00\right.$, effective samples $\left.>2.99 \times 10^{4}\right)$ revealed evidence against the interaction $(\mathrm{BF}=0.18)$. These models' posterior point summaries are reported in the Supplement Materials file available on the OSF. A one-sided $t$-test of the interaction's posterior distribution confirmed the evidence against a positive change from Before- to the After-test rating time-point: $\mathrm{BF}=0.26$. In summary, these analyses rejected the notion that participants substantially adapted their attention ratings according to their perceived performance at the test. 


\section{Table 4}

Posterior Estimates of the Effect of Predictors on Recall Error in Experiment 3. Conditions and Coefficients with Credible Gains (Positive Sign) and Detriments (Negative Sign) on Recall are in Boldface.

\begin{tabular}{|c|c|c|c|c|c|c|}
\hline \multirow{3}{*}{ Predictor } & \multicolumn{6}{|c|}{ Data } \\
\hline & \multicolumn{2}{|c|}{ All Data } & \multicolumn{2}{|c|}{ Rating Before } & \multicolumn{2}{|c|}{ Rating After } \\
\hline & $\mathbf{M}$ & 95\% HDI & $\mathbf{M}$ & $95 \%$ HDI & $\mathbf{M}$ & $95 \% \mathrm{HDI}$ \\
\hline Intercept & 0.08 & {$[-0.05,0.23]$} & -0.25 & {$[-0.41,-0.09]$} & 0.11 & {$[-0.04,0.25]$} \\
\hline Attention (+) & 0.46 & {$[0.33,0.61]$} & 0.27 & {$[0.11,0.43]$} & 0.47 & {$[0.34,0.62$} \\
\hline Valid (+) & 1.01 & {$[0.86,1.16]$} & 1.19 & {$[1.02,1.37]$} & 1.01 & {$[0.86,1.17]$} \\
\hline Invalid (-) & -1.92 & {$[-2.65,-1.36]$} & -1.21 & {$[-1.87,-0.68]$} & -2.23 & {$[-3.27,-1.45]$} \\
\hline Before (-) & -0.35 & {$[-0.58,-0.12]$} & -- & -- & & -- \\
\hline Attention $\times$ Valid $(-\&+)$ & -0.16 & {$[-0.32,-0.01]$} & 0.19 & {$[0.01,0.37]$} & -0.15 & {$[-0.32,0.01]$} \\
\hline Attention $\times$ Invalid & -0.21 & {$[-0.64,0.23]$} & 0.12 & {$[-0.28,0.54]$} & -0.21 & {$[-0.76,0.34]$} \\
\hline Attention $\times$ Before & -0.17 & {$[-0.37,0.02]$} & & & & \\
\hline Invalid $\times$ Before & 0.53 & {$[-0.15,1.24]$} & -- & -- & -- & -- \\
\hline Valid $\times$ Before & 0.21 & {$[-0.02,0.44]$} & -- & -- & -- & -- \\
\hline Attention $\times$ Invalid $\times$ Before & 0.38 & {$[-0.23,1.01]$} & -- & -- & -- & -- \\
\hline Attention $\times$ Valid $\times$ Before $(+)$ & 0.33 & {$[0.19,0.57]$} & -- & -- & -- & -- \\
\hline
\end{tabular}

Note. (+) denotes performance gains, and (-) denotes performance detriments. The regression weights reflect the change in the concentration parameter $\kappa$ of the von Mises distribution. Larger values of $\kappa$ indicate a narrower distribution and, accordingly, higher recall precision. The values are on the logarithmic scale. $\mathrm{M}=$ Mean, HDI $=$ Highest Density Interval. 


\section{Discussion}

Experiment 3 used invalid cueing to more rigorously test whether attention ratings are affected by performance evaluation. We also replicated several aspects of Experiment 2 by asking one group of participants to place their ratings before the memory test, and one group to place their ratings after the memory test. Note that cue validity is not apparent to the participant until the test item's presentation. Attention ratings were higher in both valid and invalid retro-cue conditions when requested prior to the test. A separate analysis of post-test ratings revealed a more ambiguous state of affairs: Whereas attention in valid-cue condition was rated higher than in trials with no-cues, this was not the case with the invalid cues. Ratings in invalid retro-cue trials were not as high as in the valid retro-cue condition, but they did not drop below the values observed in the no-cue condition, although invalid-cue condition yielded much worse performance. Most critically, the diagnostic data in this study are provided by the correlation of test performance and attention as a function of rating before- or after-test. With a one-sided $t$ test, we were able to reject the hypothesis that the correlation between participants' performance and attention was stronger after the test than before it.

\section{Experiment 4}

In Experiment 3, an interaction between sustained and selective attention emerged in one subset of the data (before-test rating time-point). Due to several differences between this study and the previous ones, it is difficult to gauge the robustness of this finding. Hence, we replicated Experiment 2 in an online setting similar to Experiment 3, aiming to clarify whether evidence for the independence of sustained and focused attention, as observed before, will hold. 


\section{Participants}

Participants were English speakers, resident in the UK, U.S., or Australia $(N=98$, $M_{\text {Age }}=26.55, \mathrm{SD}=5.32 ; 50$ women, 46 men, and 2 other) recruited for an online study via the Prolific platform. The study lasted $30 \mathrm{~min}$ and participants were compensated with 4.5 British Pounds. We collected the data in two batches, starting from a sample of $n=60$ and collected a further sample of $n=40$ in the second batch (datasets from one participant in each batch were lost due to a server transmission error), resulting in a total $N=98$.

\section{Procedure}

The experiment was programmed in lab.js (Henninger et al., 2020). The design was identical to Experiment 2, with only minor modifications. In one 30-minute session, participants completed 200 trials across 4 blocks with self-paced breaks in between. Retro-cues (100\% valid) appeared on $50 \%$ of the trials, randomly intermixed with NoCue Short trials. Attention ratings were solicited on $24 \%$ of the trials (i.e., 48 probes), balanced between cue conditions. As in Experiment 2, half of the attention probes appeared before the memory test and other half after it, resulting in 12 attention ratings per design cell (within-subjects manipulation). The stimuli parameters and presentation timing were identical to Experiment 3. As in Experiment 3 attention ratings were skewed to the left, we modified the instructions to strongly emphasize our interest in participants' genuine attention experience, and the unconditionality of their payment on these ratings. Reminders about this information appeared also in the breaks between the blocks. This was successful in reducing the skewness of the attention ratings in Experiment 4 (see Figure 7). 
We inspected the data for outliers using the same procedure as in Experiment 3. There were $n=4$ participants with a mean recall error $>80^{\circ}$ and $n=1$ participant only reported one attention rating value throughout the experiment (rating $S D=0$ ); none of the participants met both criteria. The analyses were conducted on both the whole sample and without the outlier participants, and we report the results for the latter. If not otherwise indicated, the results' pattern did not differ.

\section{Results}

\section{Memory Accuracy}

Recall data are visualized in Figure 8A. A 2 (Cue, No-Cue) $\times 3$ (Rating: Before, After, None) BANOVA yielded largest support for the full model with two main effects and their interaction (see Table 5 for BFs and ratios of evidence for each predictor). Similar to Experiment 2, the interaction was due to recall being statistically indistinguishable with cueing regardless of the rating time-point ( $t$-tests: No Rating vs. Rating Before, $\mathrm{BF}=0.35$; No Rating vs. Rating After, $\mathrm{BF}=0.12$; Rating Before vs. Rating After, $\mathrm{BF}=0.16)$. Without cueing, performance was comparable in trials with ratings placed after the test and trials without ratings $(\mathrm{BF}=0.32)$. But, recall was worse when ratings were solicited before the VWM test compared to after it $\left(\mathrm{BF}=2.69 \times 10^{7}\right)$, as well as compared to no-rating trials $\left(\mathrm{BF}=4.70 \times 10^{7}\right)$. In sum, placing attention ratings before the memory test hindered recall in no-cue trials, but retro-cueing protected memory representations from this interference, replicating Experiments 1, 2, and 3. 


\section{Figure 8}

Experiment 4 Results Showing Cueing Effects on Recall Error (Panel A) and Attention Ratings (Panel B) as Well as Displaying a Constant Retro-Cue Benefit over All Levels of Self-Rated Attention (Panel C Presents Observed Data and Panel D the Model Predictions).

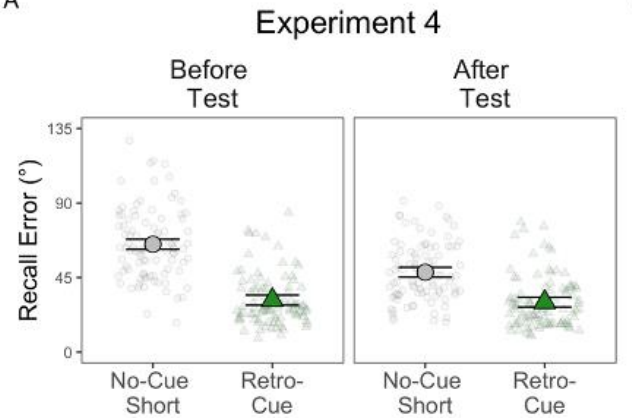

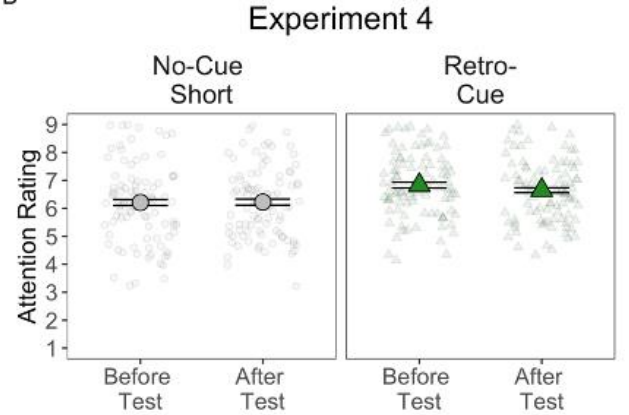

C

Experiment 4

Data

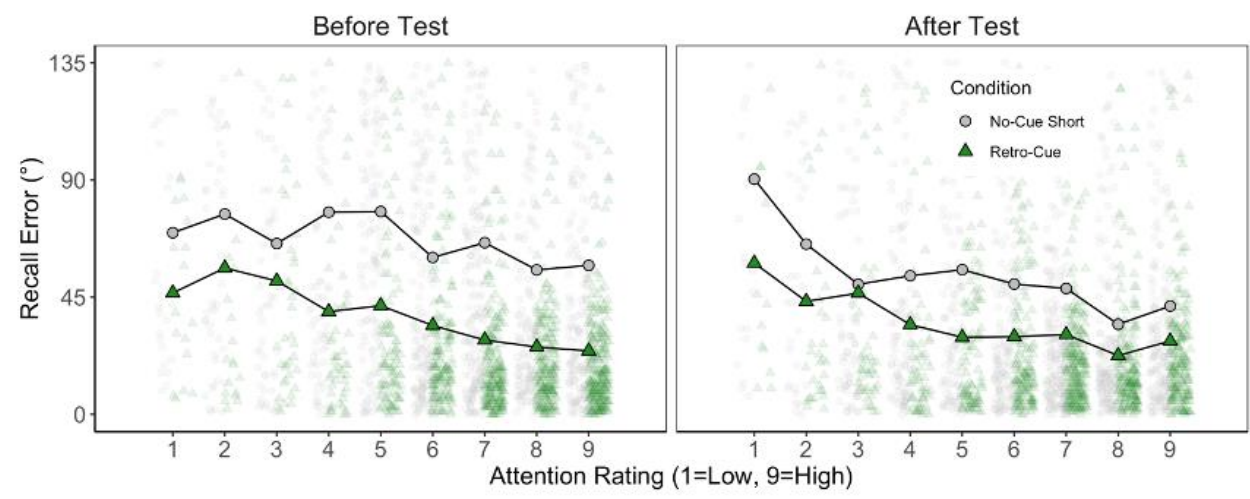

D

Experiment 4

Predictions

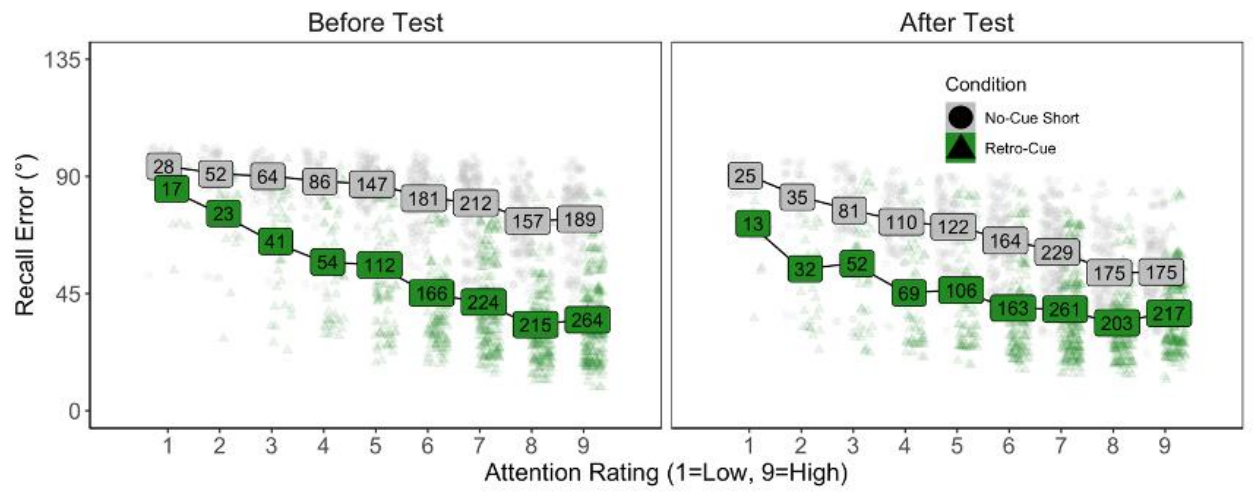

Note. Small dots in Panels A and B are each individual's average in that condition, and the large dots are the sample mean. Error bars are the $95 \%$ within-subjects' confidence intervals. In Panels $\mathrm{C}$ and D, the dot clouds are the individual observations. Panel D displays the number of observations entered in the model per each condition combination level. The data are from the subsample $(N=93)$ without outlier participants. 


\section{Table 5}

Results of the BANOVA on the Recall Error and on the Attention Rating Data in Experiment 4 Showing Credible Effects of All Predictors on Recall Error, But Only Cue Effects on Attention Ratings.

\begin{tabular}{|c|c|c|c|c|}
\hline \multirow[t]{2}{*}{ Model } & \multicolumn{2}{|c|}{ Recall Error } & \multicolumn{2}{|c|}{ Attention Ratings } \\
\hline & Model & Predictor & Model & Predictor \\
\hline Cue & $2.70 \times 10^{66}$ & $1.63 \times 10^{72}$ & $3.22 \times 10^{17}$ & $4.44 \times 10^{17}$ \\
\hline Rating Time & $9.70 \times 10^{3}$ & $5.85 \times 10^{9}$ & 0.26 & 0.37 \\
\hline Cue + Rating Time & $1.58 \times 10^{76}$ & --- & $1.99 \times 10^{17}$ & --- \\
\hline Cue + Rating Time + Cue $\times$ Rating Time & $2.86 \times 10^{83}$ & $1.91 \times 10^{7}$ & $1.07 \times 10^{17}$ & 0.89 \\
\hline
\end{tabular}

Note. The Model-column presents the evidence (BF) for the specified model against the Null. The Predictor-column presents the evidence for the inclusion of the factor printed in italics when contrasted to the two main effects' model (credible effects, i.e., BFs > 3 are printed in bold). The reported analyses were conducted on the data without outlier participants.

\section{Attention Ratings}

Attention rating data are visualized in Figure 8B (see Figure 7 for the frequency of observations per rating level in each condition). A 2 (Cue, No-Cue) $\times 2$ (Rating: Before, After) BANOVA yielded largest support for the model with only the main effect of cueing (see Table 5). Thus, similar to Experiment 2, retro-cueing increased participants' attention irrespective of the rating time-point. 


\section{Joint Effects of Attention Rating and Cueing on Accuracy}

Addressing the main question of interrelation of sustained and selective attention, we modelled the effects of cueing, self-rated attention levels and rating time-point on response deviation using the same approach and settings as in Experiment 2 (see Figure $8 \mathrm{C}$ for the visualization of the data and $8 \mathrm{D}$ for model's predictions). We used the full scale of attention ratings (1-9), because the data was more normally distributed in Experiment 4, and ran the models with and without the excluded participants; the results' pattern did not differ (see Table S7 in the Supplement for model coefficients with the full sample). The model showed an adequate fit (for population-level effects, $\hat{R}=1.00$, effective samples $>2161$ ). Posterior distributions are summarized in Table 1 . The results replicated Experiment 2: There were credible main effects of cueing and attention, showing an improvement of recall precision. Placing attention ratings prior to memory test had a negative effect on recall precision. However, this disadvantage was mitigated by the cueing procedure, as evidenced by a credible two-way interaction between Rating Before test time-point and Retro-Cue. Most crucially, we did not observe a credible interaction between valid cueing and attention, as the posterior distribution of this effect included zero. This result corroborates our previous observations of independent effects of sustained and selective attention in VWM.

Lastly, we evaluated the directional hypothesis that the correlation between attention ratings and performance increased from the Before-Test to After-Test time point, which would show that attention appraisals were influenced by behavioral performance evaluation. Similar to Experiment 3, we again computed two models: one with only the main effects of Attention and Rating Time-Point, and another with the main 
effects and their interaction (the models and full procedure are reported in the Supplement). Directional $t$-test provided evidence against the hypothesis that performance and rated attention would be more correlated after memory test than before $(\mathrm{BF}=0.03)$. In sum, this result parallels our finding from Experiment 3 which also demonstrated that participants did not substantially alter their attention appraisals to match their perceived performance.

\section{Discussion}

Using the same design as Experiment 2, Experiment 4 replicated the independent effects of sustained and focused attention in a larger sample and in the online setting, again yielding linear performance improvements with higher self-rated sustained attention and an additive benefit from retro-cueing.

One possibility is that the credible interaction observed in the Before-Test rating subset of Experiment 3 may represent a false positive, especially as there were issues with restricted attention ratings' variance in this online sample. Another possibility is that the interaction in Experiment 3 reflects participants' strategic choice to invest focused attention in the cue or not, which they incorporated in their attention ratings. When ratings were solicited before the test, participants did not know if the cue will be valid, but were instructed that some cues may not. Attending to invalid cues conveys performance costs as non-attended items may be removed from VWM; although this is reversible (Lewis-Peacock et al., 2018), participants may prefer to avoid the cost by discounting the cue instead. Thus, on some cue trials, participants may have elected to not focus on the cued item, and they may have incorporated their degree of trust in the cue in their attention ratings. This would explain why the retro-cue benefit increased with 
higher rated attention in the Before-Test group of Experiment 3: If in the instances when attention was rated as high, participants invested more focused attention on the cued item, this would result in a larger benefit compared to other trials when participants chose not to use the cue.

\section{General Discussion}

The present study examined the interplay between sustained and selective attention in VWM. Specifically, we tested if performance improvements from selective attention in VWM are conditional on the state of sustained attention. We assessed the covariation of self-rated attention, retro-cueing and memory accuracy in a continuous color reproduction task. In four experiments, we observed independent effects of selfrated attention and cueing on memory accuracy for six (E1, E2 probed before- and aftertest, E3 after-test, E4 before- and after-test) out of seven comparisons. This suggests that the benefit of focusing selective attention in VWM generally occurs independently from the level of sustained attention. Converging with our results, recent research observed similar independent contributions of sustained and selective attention on long-term memory (LTM) performance for continuous features (deBettencourt et al., 2020).

In the following sections, we will discuss the implications of our findings for the assessment of the role of attention in VWM.

\section{The Role of Attention for Encoding, Maintenance and Retrieval}

Attention and WM are closely entwined cognitive processes (Chun, 2011; Gazzaley \& Nobre, 2012; Kiyonaga \& Egner, 2013; Oberauer, 2019). Attention is assumed to be required for VWM encoding, maintenance, and retrieval. It remains uncharted area how the role of attention in VWM relates to different modes of attention 
as, for example, sustained attention on the one hand, and selective focused attention on the other hand.

Both selective and sustained attention seem to be relevant for the encoding of information in VWM. Encoding into VWM is unsuccessful if selective attention is still engaged by the processing of a target that appeared very shortly before, as is evident from the attentional blink effect (Dux \& Marois, 2009; Martens \& Wyble, 2010; Ricker \& Hardman, 2017). Furthermore, information that is selectively attended at encoding (e.g., through a pre-cue) receives priority for entering VWM (Astle et al., 2012; Schmidt et al., 2002; Souza, Thalmann, et al., 2018). Relatedly, spontaneous fluctuations of sustained attention also impact VWM encoding (Adam \& Vogel, 2017; deBettencourt et al., 2018, 2019, 2020; Killebrew et al., 2018).

Similarly, maintenance of VWM representations is assumed to require active sustained attention (Awh et al., 2006; Chun, 2011; Cowan, 1998; Gazzaley \& Nobre, 2012; Jonides et al., 2008; Kiyonaga \& Egner, 2013; Logie, 2014; C. C. Morey \& Bieler, 2013; Olivers, 2008; Theeuwes et al., 2009). In VWM tasks, participants are required to maintain arrays of several memory items, which demands the distribution of attention across them (Makovski \& Jiang, 2007). Imposing tasks that demand attention during the retention interval hinders VWM performance (Allen et al., 2006; Brown \& Brockmole, 2010; C. C. Morey \& Bieler, 2013), and more so the more attentionally demanding the task is (Loaiza \& Souza, 2019; Souza \& Oberauer, 2017; Vergauwe et al., 2014; but see Ricker \& Vergauwe, 2020). However, the notion that sustained attention is necessary for VWM maintenance is being challenged by other studies demonstrating that performance decrements occur in some dual-task conditions but not others (Hollingworth \& Maxcey- 
Richard, 2013; Lewis-Peacock et al., 2011; Olivers et al., 2011; Rerko et al., 2014; van Moorselaar, Olivers, et al., 2015; Wang et al., 2018, 2019). Disruption of VWM maintenance is observed when the secondary task requires central attention, but not when only visual attention is engaged. For example, Souza and Oberauer (2017) observed that engaging sustained visual attention during the retention interval by requiring participants to monitor a flicker in the center of the screen had a negligible impact on VWM recall. Instead, engaging central attention by requesting the decision of whether a sequence of one or two tones was high or low substantially impacted performance, and this cost was higher the longer attention was engaged by the task. Therefore, VWM maintenance may depend on some forms of attention, such as selective attention, but not others such as sustained attention.

A further hypothesis is that attention is necessary for consolidation of memory i.e., the transfer of a representation into a robust state in VWM (Ricker et al., 2018), and disruption from intervening tasks is negligible after consolidation has been completed. Wang et al. (2019) found that disruption of memory for a colored grating peaked when an intervening letter change-detection task occurred $250 \mathrm{~ms}$ after the offset of the memory object, but not earlier (i.e., $0 \mathrm{~ms}$ ) or later (i.e., 500 and $1000 \mathrm{~ms}$ ). According to these results, attention may serve the consolidation of a memory trace, rather than its maintenance.

At the retrieval stage, attentional selection is necessary to ensure the correct retrieval of the currently tested information. Retro-cues are assumed to benefit memory as they allow for retrieval to commence even before the onset of the test array, giving a retrieval "head start". Retrieving a representation in advance of the test helps to bypass 
the visual interference from the test display as well as interference from decision-making processes (Shepherdson et al., 2018; Souza et al., 2016; Tabi et al., 2019).

In a nutshell, the role of sustained attention has been mostly emphasized for the encoding and maintenance stages of VWM. In contrast to that, retro-cue effects have been discussed in relation to the maintenance and retrieval stages. This suggests that these two forms of attention may be seen as required in different phases of the VWM trial, explaining why their joint effect has been hardly explored. Our observation of independent effects of sustained and selective attention in VWM may well indicate that they are operating at different stages of the VWM trial.

\section{Assessing Attentional Fluctuations in VWM}

Electrophysiological evidence suggests that human sustained attention fluctuations follow a rhythmic pattern (Helfrich et al., 2018; Kam et al., 2011), which has been subsumed in a neural model of sustained attention (Clayton et al., 2015). However, there is still uncertainty about the time frame in which such fluctuations can be expected and how they impact VWM performance. In the SART literature, variability in response time across several trials is a sensitive indicator of sustained on-task attention. Response time variability measures predict errors in the SART (Robertson et al., 1997) as well as VWM accuracy in a SART-VWM hybrid task (deBettencourt et al., 2018, 2019), and correlate with self-rated on-task focus versus mind-wandering reports (reviewed in Randall et al., 2014).

Instead of prospectively classifying sustained attention levels from reaction times (as in deBettencourt et al., 2019), we measured attention self-ratings, which is another viable approach to assess attentional states in VWM (Adam \& Vogel, 2017; Arnicane et 
al., 2021). Our main goal was to evaluate the interdependency of sustained vs. selective attention, which we observed based on the covariation of self-rated attention and performance. However, given that attention ratings were placed around the time of retrieval (either before or after the test), it is difficult to disentangle in which timepoint during the trial attention fluctuations occurred.

Our results suggest that the maintenance stage did not produce substantial changes in attention (see Experiment 1), leaving the encoding and retrieval stages as more relevant candidates for the locus of the variability in attentional states. Namely, Experiment 1 demonstrated that, without retro-cues, longer storage interval (2.25 s) was not associated with changes in self-rated attention compared to a shorter ( $1 \mathrm{~s})$ one. Future studies are needed to assess whether attention fluctuations contribute to maintenance across longer retention intervals, which can be particularly relevant for testing proposals that VWM maintenance involves sustained visual attention to representations (Chun, 2011). Time-sensitive measures such as EEG could also be useful to disentangle the time period in which fluctuations occurred (Killebrew et al., 2018; Mayer et al., 2020), as reflected in the self-reports of low or high-levels of attention.

\section{Conclusion}

Our main finding was that the size of the retro-cue benefit did not depend on the level of self-rated attention. Although attentional states fluctuate across trials leading to visual information being encoded with varying quality, selective focused attention can be retrospectively applied to these varying-quality VWM representations, providing a steady-increment benefit. This finding attests to the flexibility of VWM and the multifaceted roles that attention plays in this system. Arguably, sustained attention may 
contribute to the uptake of perceptual information that can be translated to a robust VWM representation, whereas selective focused attention operating at the maintenance and retrieval stages (as indexed by the retro-cue effect) selects among these representations for recall.

The independent effects of sustained and focused attention suggest that either these two modes of attention operate by independent mechanisms, or they may operate in separate phases of the VWM task, thus minimizing their interaction and leading to their observed additive contributions to performance. 


\section{References}

Adam, K. C. S., Mance, I., Fukuda, K., \& Vogel, E. K. (2015). The Contribution of Attentional Lapses to Individual Differences in Visual Working Memory Capacity. Journal of Cognitive Neuroscience, 27(8), 1601-1616. https://doi.org/10.1162/jocn_a_00811

Adam, K. C. S., \& Vogel, E. K. (2017). Confident failures: Lapses of working memory reveal a metacognitive blind spot. Attention, Perception, \& Psychophysics, 79(5), 1506-1523. https://doi.org/10.3758/s13414-017-1331-8

Allen, R. J., Baddeley, A. D., \& Hitch, G. J. (2006). Is the binding of visual features in working memory resource-demanding? Journal of Experimental Psychology: General, 135(2), 298-313. https://doi.org/10.1037/0096-3445.135.2.298

Arnicane, A., Oberauer, K., \& Souza, A. S. (2021). Validity of attention self-reports in younger and older adults. Cognition, 206, 104482. https://doi.org/10.1016/j.cognition.2020.104482

Astle, D. E., Summerfield, J., Griffin, I., \& Nobre, A. C. (2012). Orienting attention to locations in mental representations. Attention, Perception, \& Psychophysics, 74(1), 146-162. https://doi.org/10.3758/s13414-011-0218-3

Awh, E., Vogel, E. K., \& Oh, S.-H. (2006). Interactions between attention and working memory. Neuroscience, 139(1), 201-208. https://doi.org/10.1016/j.neuroscience.2005.08.023

Berryhill, M. E., Richmond, L. L., Shay, C. S., \& Olson, I. R. (2012). Shifting attention among working memory representations: Testing cue type, awareness, and 
strategic control. The Quarterly Journal of Experimental Psychology, 65(3), 426438.

Brainard, D. H. (1997). The psychophysics toolbox. Spatial Vision, 10(4), 433-436.

Brown, L. A., \& Brockmole, J. R. (2010). The role of attention in binding visual features in working memory: Evidence from cognitive ageing. The Quarterly Journal of Experimental Psychology, 63(10), 2067-2079.

https://doi.org/10.1080/17470211003721675

Bürkner, P.-C. (2018). Advanced Bayesian Multilevel Modeling with the R Package brms. The R Journal, 10(1), 395-411. https://doi.org/doi.org/10.32614/RJ-2018017

Chun, M. M. (2011). Visual working memory as visual attention sustained internally over time. Neuropsychologia, 49(6), 1407-1409. https://doi.org/10.1016/j.neuropsychologia.2011.01.029

Chun, M. M., Golomb, J. D., \& Turk-Browne, N. B. (2011). A Taxonomy of External and Internal Attention. Annual Review of Psychology, 62(1), 73-101. https://doi.org/10.1146/annurev.psych.093008.100427

Clayton, M. S., Yeung, N., \& Cohen Kadosh, R. (2015). The roles of cortical oscillations in sustained attention. Trends in Cognitive Sciences, 19(4), 188-195. https://doi.org/10.1016/j.tics.2015.02.004

Cowan, N. (1998). Attention and Memory: An integrated framework. Oxford University Press. 
Cowan, N. (2010). The Magical Mystery Four: How Is Working Memory Capacity Limited, and Why? Current Directions in Psychological Science, 19(1), 51-57. https://doi.org/10.1177/0963721409359277

Cowan, N. (2011). The focus of attention as observed in visual working memory tasks: Making sense of competing claims. Neuropsychologia, 49(6), 1401-1406. https://doi.org/10.1016/j.neuropsychologia.2011.01.035

Curran, P. G. (2016). Methods for the detection of carelessly invalid responses in survey data. Journal of Experimental Social Psychology, 66, 4-19. https://doi.org/10.1016/j.jesp.2015.07.006

deBettencourt, M. T., Keene, P. A., Awh, E., \& Vogel, E. K. (2019). Real-time triggering reveals concurrent lapses of attention and working memory. Nature Human Behaviour, 3(8), 808-816. https://doi.org/10.1038/s41562-019-0606-6

deBettencourt, M. T., Norman, K. A., \& Turk-Browne, N. B. (2018). Forgetting from lapses of sustained attention. Psychonomic Bulletin \& Review, 25(2), 605-611. https://doi.org/10.3758/s13423-017-1309-5

deBettencourt, M. T., Williams, S. D., Vogel, E. K., \& Awh, E. (2020). Sustained attention and spatial attention distinctly influence long-term memory encoding. BioRxiv, 2020.09.14.297341. https://doi.org/10.1101/2020.09.14.297341

Dux, P. E., \& Marois, R. (2009). The attentional blink: A review of data and theory. Attention, Perception, \& Psychophysics, 71(8), 1683-1700. https://doi.org/10.3758/APP.71.8.1683

Esterman, M., \& Rothlein, D. (2019). Models of sustained attention. Current Opinion in Psychology, 29, 174-180. https://doi.org/10.1016/j.copsyc.2019.03.005 
Fukuda, K., Woodman, G. F., \& Vogel, E. K. (2015). Individual Differences in Visual Working Memory Capacity. In Mechanisms of Sensory Working Memory (pp. 105-119). Elsevier. https://doi.org/10.1016/B978-0-12-801371-7.00009-0

Gazzaley, A., \& Nobre, A. C. (2012). Top-down modulation: Bridging selective attention and working memory. Trends in Cognitive Sciences, 16(2), 129-135. https://doi.org/10.1016/j.tics.2011.11.014

Gözenman, F., Tanoue, R. T., Metoyer, T., \& Berryhill, M. E. (2014). Invalid retro-cues can eliminate the retro-cue benefit: Evidence for a hybridized account. Journal of Experimental Psychology: Human Perception and Performance, 40(5), 17481754. https://doi.org/10.1037/a0037474

Gressmann, M., \& Janczyk, M. (2016). The (Un)Clear Effects of Invalid Retro-Cues. Frontiers in Psychology, 7. https://doi.org/10.3389/fpsyg.2016.00244

Griffin, I. C., \& Nobre, A. C. (2003). Orienting Attention to Locations in Internal Representations. Journal of Cognitive Neuroscience, 15(8), 1176-1194. https://doi.org/10.1162/089892903322598139

Gunseli, E., Fahrenfort, J., Daoultzis, K., Meeter, M., \& Olivers, C. N. L. (2015). The Loss of Information from Visual Working Memory depends on Retro-Cue Reliability. Journal of Vision, 15(12), 91-91. https://doi.org/10.1167/15.12.91

Gunseli, E., Fahrenfort, J., Moorselaar, D. van, Daoultzis, K., Meeter, M., \& Olivers, C. N. L. (2018). Unattended but actively stored: EEG dynamics reveal a dissociation between selective attention and storage in working memory. BioRxiv, 320952. https://doi.org/10.1101/320952 
Hardman, K. O., Vergauwe, E., \& Ricker, T. J. (2017). Categorical working memory representations are used in delayed estimation of continuous colors. Journal of Experimental Psychology: Human Perception and Performance, 43(1), 30-54. https://doi.org/10.1037/xhp0000290

Helfrich, R. F., Fiebelkorn, I. C., Szczepanski, S. M., Lin, J. J., Parvizi, J., Knight, R. T., \& Kastner, S. (2018). Neural Mechanisms of Sustained Attention Are Rhythmic. Neuron, 99(4), 854-865.e5. https://doi.org/10.1016/j.neuron.2018.07.032

Henninger, F., Shevchenko, Y., Mertens, U. K., Kieslich, P. J., \& Hilbig, B. E. (2020). lab.js: A free, open, online study builder. https://doi.org/10.5281/zenodo.597045

Hollingworth, A., \& Maxcey-Richard, A. M. (2013). Selective Maintenance in Visual Working Memory Does Not Require Sustained Visual Attention. Journal of Experimental Psychology. Human Perception and Performance, 39(4), 10471058. https://doi.org/10.1037/a0030238

Jonides, J., Lewis, R. L., Nee, D. E., Lustig, C. A., Berman, M. G., \& Moore, K. S. (2008). The Mind and Brain of Short-Term Memory. Annual Review of Psychology, 59(1), 193-224. https://doi.org/10.1146/annurev.psych.59.103006.093615

Kam, J. W. Y., Dao, E., Farley, J., Fitzpatrick, K., Smallwood, J., Schooler, J. W., \& Handy, T. C. (2011). Slow Fluctuations in Attentional Control of Sensory Cortex. Journal of Cognitive Neuroscience, 23(2), 460-470. https://doi.org/10.1162/jocn.2010.21443

Killebrew, K. W., Gurariy, G., Peacock, C. E., Berryhill, M. E., \& Caplovitz, G. P. (2018). Electrophysiological correlates of encoding processes in a full-report 
visual working memory paradigm. Cognitive, Affective, \& Behavioral

Neuroscience, 18(2), 353-365. https://doi.org/10.3758/s13415-018-0574-8

Kiyonaga, A., \& Egner, T. (2013). Working memory as internal attention: Toward an integrative account of internal and external selection processes. Psychonomic Bulletin \& Review, 20(2), 228-242. https://doi.org/10.3758/s13423-012-0359-y

Landman, R., Spekreijse, H., \& Lamme, V. A. F. (2003). Large capacity storage of integrated objects before change blindness. Vision Research, 43(2), 149-164. https://doi.org/10.1016/S0042-6989(02)00402-9

Lewis-Peacock, J. A., Drysdale, A. T., Oberauer, K., \& Postle, B. R. (2011). Neural Evidence for a Distinction between Short-term Memory and the Focus of Attention. Journal of Cognitive Neuroscience, 24(1), 61-79. https://doi.org/10.1162/jocn_a_00140

Lewis-Peacock, J. A., Kessler, Y., \& Oberauer, K. (2018). The removal of information from working memory. Annals of the New York Academy of Sciences, 1424(1), 33-44. https://doi.org/10.1111/nyas.13714

Loaiza, V. M., \& Souza, A. S. (2019). An age-related deficit in preserving the benefits of attention in working memory. Psychology and Aging, 32(2), 282.

Logie, R. H. (2014). Logie, R. H., \& Logie, R. H. (1995). Visuo-spatial working memory. Lawrence Erlbaum Associates.

Luck, S. J., \& Vogel, E. K. (2013). Visual working memory capacity: From psychophysics and neurobiology to individual differences. Trends in Cognitive Sciences, 17(8), 391-400. https://doi.org/10.1016/j.tics.2013.06.006 
Makovski, T., \& Jiang, Y. V. (2007). Distributing versus focusing attention in visual short-term memory. Psychonomic Bulletin \& Review, 14(6), 1072-1078. https://doi.org/10.3758/BF03193093

Makovski, T., \& Pertzov, Y. (2015). Attention and memory protection: Interactions between retrospective attention cueing and interference. Quarterly Journal of Experimental Psychology, 68(9), 1735-1743. https://doi.org/10.1080/17470218.2015.1049623

Makovski, T., Sussman, R., \& Jiang, Y. V. (2008). Orienting attention in visual working memory reduces interference from memory probes. Journal of Experimental Psychology: Learning, Memory, and Cognition, 34(2), 369-380. https://doi.org/10.1037/0278-7393.34.2.369

Makovski, T., Watson, L. M., Koutstaal, W., \& Jiang, Y. V. (2010). Method matters: Systematic effects of testing procedure on visual working memory sensitivity. Journal of Experimental Psychology: Learning, Memory, and Cognition, 36(6), 1466-1479. https://doi.org/10.1037/a0020851

Martens, S., \& Wyble, B. (2010). The attentional blink: Past, present, and future of a blind spot in perceptual awareness. Neuroscience \& Biobehavioral Reviews, 34(6), 947-957. https://doi.org/10.1016/j.neubiorev.2009.12.005

Matsukura, M., Luck, S. J., \& Vecera, S. P. (2007). Attention effects during visual shortterm memory maintenance: Protection or prioritization? Perception \& Psychophysics, 69(8), 1422-1434. https://doi.org/10.3758/BF03192957 
Mayer, J. S., Korinth, S., Peters, B., \& Fiebach, C. J. (2020). An Electrophysiological Dissociation of Encoding vs. Maintenance Failures in Visual-Spatial Working Memory. Frontiers in Psychology, 11. https://doi.org/10.3389/fpsyg.2020.00522

Mitchell, D. J., Cusack, R., \& Cam-CAN. (2018). Visual short-term memory through the lifespan: Preserved benefits of context and metacognition. Psychology and Aging, 33(5), 841-854. https://doi.org/10.1037/pag0000265

Morey, C. C., \& Bieler, M. (2013). Visual short-term memory always requires general attention. Psychonomic Bulletin \& Review, 20(1), 163-170. https://doi.org/10.3758/s13423-012-0313-z

Morey, R. D., Rouder, J. N., Jamil, T., Forner, K., \& Ly, A. (2018). Package 'bayesfactor'. (0.9.12-4.2) [Computer software]. https://richarddmorey.github.io/BayesFactor/

Mrazek, M. D., Smallwood, J., Franklin, M. S., Chin, J. M., Baird, B., \& Schooler, J. W. (2012). The role of mind-wandering in measurements of general aptitude. Journal of Experimental Psychology: General, 141(4), 788-798. https://doi.org/10.1037/a0027968

Myers, N. E., Stokes, M. G., \& Nobre, A. C. (2017). Prioritizing Information during Working Memory: Beyond Sustained Internal Attention. Trends in Cognitive Sciences, 21(6), 449-461. https://doi.org/10.1016/j.tics.2017.03.010

Oberauer, K. (2009). Chapter 2 Design for a Working Memory. In Psychology of Learning and Motivation (Vol. 51, pp. 45-100). Academic Press. https://doi.org/10.1016/S0079-7421(09)51002-X 
Oberauer, K. (2013). The focus of attention in working memory-From metaphors to mechanisms. Frontiers in Human Neuroscience, 7. https://doi.org/10.3389/fnhum.2013.00673

Oberauer, K. (2019). Working Memory and Attention - A Conceptual Analysis and Review. Journal of Cognition, 2(1), 36. https://doi.org/10.5334/joc.58

Olivers, C. N. L. (2008). Interactions between visual working memory and visual attention. Frontiers in Bioscience, 13(3), 1182-1191.

Olivers, C. N. L., Peters, J., Houtkamp, R., \& Roelfsema, P. R. (2011). Different states in visual working memory: When it guides attention and when it does not. Trends in Cognitive Sciences, 15(7), 327-334. https://doi.org/10.1016/j.tics.2011.05.004

Pelli, D. G. (1997). The VideoToolbox software for visual psychophysics: Transforming numbers into movies. Spatial Vision, 10(4), 437-442.

Pertzov, Y., Bays, P. M., Joseph, S., \& Husain, M. (2013). Rapid forgetting prevented by retrospective attention cues. Journal of Experimental Psychology: Human Perception and Performance, 39(5), 1224-1231. https://doi.org/10.1037/a0030947

Peters, B., Rahm, B., Kaiser, J., \& Bledowski, C. (2019). Differential trajectories of memory quality and guessing across sequential reports from working memory. Journal of Vision, 19(7), 3-3. https://doi.org/10.1167/19.7.3

Prinzmetal, W., Amiri, H., Allen, K., \& Edwards, T. (1998). Phenomenology of attention: I Color, location, orientation, and spatial frequency. Journal of Experimental Psychology: Human Perception and Performance, 24(1), 261-282. https://doi.org/10.1037/0096-1523.24.1.261 
R Core Team. (2018). R: A language and environment for statistical computing. $R$ Foundation for Statistical Computing, Vienna, Austria. https://www.Rproject.org/.

Rademaker, R. L., Tredway, C. H., \& Tong, F. (2012). Introspective judgments predict the precision and likelihood of successful maintenance of visual working memory. Journal of Vision, 12(13), 21-21. https://doi.org/10.1167/12.13.21

Randall, J. G., Oswald, F. L., \& Beier, M. E. (2014). Mind-wandering, cognition, and performance: A theory-driven meta-analysis of attention regulation. Psychological Bulletin, 140(6), 1411-1431. https://doi.org/10.1037/a0037428

Rerko, L., \& Oberauer, K. (2013). Focused, unfocused, and defocused information in working memory. Journal of Experimental Psychology: Learning, Memory, and Cognition, 39(4), 1075-1096. https://doi.org/10.1037/a0031172

Rerko, L., Souza, A. S., \& Oberauer, K. (2014). Retro-cue benefits in working memory without sustained focal attention. Memory \& Cognition, 42(5), 712-728. https://doi.org/10.3758/s13421-013-0392-8

Ricker, T. J., \& Hardman, K. O. (2017). The nature of short-term consolidation in visual working memory. Journal of Experimental Psychology: General, 146(11), 15511573. https://doi.org/10.1037/xge0000346

Ricker, T. J., \& Vergauwe, E. (2020). Consistent Failure to Produce a Cognitive Load Effect in Visual Working Memory Using a Standard Dual-Task Procedure. Journal of Cognition, 3(1). https://doi.org/10.5334/joc.108 
Robertson, I. H., Manly, T., Andrade, J., Baddeley, B. T., \& Yiend, J. (1997). Oops!':

Performance correlates of everyday attentional failures in traumatic brain injured and normal subjects. Neuropsychologia, 35(6), 747-758.

Rouder, J. N. (2014). Optional stopping: No problem for Bayesians. Psychonomic Bulletin \& Review, 21(2), 301-308. https://doi.org/10.3758/s13423-014-0595-4

Rouder, J. N., Morey, R. D., Cowan, N., Zwilling, C. E., Morey, C. C., \& Pratte, M. S. (2008). An assessment of fixed-capacity models of visual working memory. Proceedings of the National Academy of Sciences, 105(16), 5975-5979. https://doi.org/10.1073/pnas.0711295105

Rummel, J., \& Boywitt, C. D. (2014). Controlling the stream of thought: Working memory capacity predicts adjustment of mind-wandering to situational demands. Psychonomic Bulletin \& Review, 21(5), 1309-1315. https://doi.org/10.3758/s13423-013-0580-3

Sahar, T., Sidi, Y., \& Makovski, T. (2020). A Metacognitive Perspective of Visual Working Memory With Rich Complex Objects. Frontiers in Psychology, 11. https://doi.org/10.3389/fpsyg.2020.00179

Samaha, J., \& Postle, B. R. (2017). Correlated individual differences suggest a common mechanism underlying metacognition in visual perception and visual short-term memory. Proceedings of the Royal Society B: Biological Sciences, 284(1867), 20172035. https://doi.org/10.1098/rspb.2017.2035

Schmidt, B. K., Vogel, E. K., Woodman, G. F., \& Luck, S. J. (2002). Voluntary and automatic attentional control of visual working memory. Perception \& Psychophysics, 64(5), 754-763. https://doi.org/10.3758/BF03194742 
Shepherdson, P., Oberauer, K., \& Souza, A. S. (2018). Working memory load and the retro-cue effect: A diffusion model account. Journal of Experimental Psychology: Human Perception and Performance, 44(2), 286-310. https://doi.org/10.1037/xhp0000448

Sligte, I. G., Scholte, H. S., \& Lamme, V. A. F. (2008). Are There Multiple Visual ShortTerm Memory Stores? PLOS ONE, 3(2), e1699. https://doi.org/10.1371/journal.pone.0001699

Smallwood, J., \& Schooler, J. W. (2006). The restless mind. Psychological Bulletin, 132(6), 946-958. https://doi.org/10.1037/0033-2909.132.6.946

Souza, A. S., \& Oberauer, K. (2016). In search of the focus of attention in working memory: 13 years of the retro-cue effect. Attention, Perception, \& Psychophysics, 78(7), 1839-1860. https://doi.org/10.3758/s13414-016-1108-5

Souza, A. S., \& Oberauer, K. (2017). The contributions of visual and central attention to visual working memory. Attention, Perception, \& Psychophysics, 79(7), 18971916. https://doi.org/10.3758/s13414-017-1357-y

Souza, A. S., Rerko, L., Lin, H.-Y., \& Oberauer, K. (2014). Focused attention improves working memory: Implications for flexible-resource and discrete-capacity models. Attention, Perception, \& Psychophysics, 76(7), 2080-2102. https://doi.org/10.3758/s13414-014-0687-2

Souza, A. S., Rerko, L., \& Oberauer, K. (2014). Unloading and reloading working memory: Attending to one item frees capacity. Journal of Experimental Psychology: Human Perception and Performance, 40(3), 1237-1256. https://doi.org/10.1037/a0036331 
Souza, A. S., Rerko, L., \& Oberauer, K. (2016). Getting more from visual working memory: Retro-cues enhance retrieval and protect from visual interference. Journal of Experimental Psychology: Human Perception and Performance, 42(6), 890-910. https://doi.org/10.1037/xhp0000192

Souza, A. S., Thalmann, M., \& Oberauer, K. (2018). The precision of spatial selection into the focus of attention in working memory. Psychonomic Bulletin \& Review, 25(6), 2281-2288. https://doi.org/10.3758/s13423-018-1471-4

Souza, A. S., Vergauwe, E., \& Oberauer, K. (2018). Where to attend next: Guiding refreshing of visual, spatial, and verbal representations in working memory. Annals of the New York Academy of Sciences, 1424(1), 76-90. https://doi.org/10.1111/nyas.13621

Tabi, Y. A., Husain, M., \& Manohar, S. G. (2019). Recall cues interfere with retrieval from visuospatial working memory. British Journal of Psychology, 110(2), 288305. https://doi.org/10.1111/bjop.12374

Theeuwes, J., Belopolsky, A., \& Olivers, C. N. L. (2009). Interactions between working memory, attention and eye movements. Acta Psychologica, 132(2), 106-114. https://doi.org/10.1016/j.actpsy.2009.01.005

Unsworth, N., \& McMillan, B. D. (2014a). Trial-to-trial fluctuations in attentional state and their relation to intelligence. Journal of Experimental Psychology: Learning, Memory, and Cognition, 40(3), 882-891. https://doi.org/10.1037/a0035544

Unsworth, N., \& McMillan, B. D. (2014b). Fluctuations in pre-trial attentional state and their influence on goal neglect. Consciousness and Cognition, 26, 90-96. https://doi.org/10.1016/j.concog.2014.03.003 
Unsworth, N., \& Robison, M. K. (2016). The influence of lapses of attention on working memory capacity. Memory \& Cognition, 44(2), 188-196. https://doi.org/10.3758/s13421-015-0560-0

van den Berg, R., Yoo, A. H., \& Ma, W. J. (2017). Fechner's law in metacognition: A quantitative model of visual working memory confidence. Psychological Review, 124(2), 197-214. https://doi.org/10.1037/rev0000060

van Moorselaar, D., Gunseli, E., Theeuwes, J., \& Olivers, C. N. L. (2015). The time course of protecting a visual memory representation from perceptual interference. Frontiers in Human Neuroscience, 8. https://doi.org/10.3389/fnhum.2014.01053 van Moorselaar, D., Olivers, C. N. L., Theeuwes, J., Lamme, V. A. F., \& Sligte, I. G. (2015). Forgotten but not gone: Retro-cue costs and benefits in a double-cueing paradigm suggest multiple states in visual short-term memory. Journal of Experimental Psychology: Learning, Memory, and Cognition, 41(6), 1755-1763. https://doi.org/10.1037/xlm0000124

Vandenbroucke, A. R. E., Sligte, I. G., Barrett, A. B., Seth, A. K., Fahrenfort, J. J., \& Lamme, V. A. F. (2014). Accurate Metacognition for Visual Sensory Memory Representations. Psychological Science, 25(4), 861-873. https://doi.org/10.1177/0956797613516146

Vergauwe, E., Langerock, N., \& Barrouillet, P. (2014). Maintaining information in visual working memory: Memory for bindings and memory for features are equally disrupted by increased attentional demands. Canadian Journal of Experimental Psychology/Revue Canadienne de Psychologie Expérimentale, 68(3), 158-162. https://doi.org/10.1037/cep0000025 
Wagenmakers, E., Wetzels, R., Borsboom, D., \& van der Maas, H. L. J. (2011). Why psychologists must change the way they analyze their data: The case of psi: Comment on Bem (2011). Journal of Personality and Social Psychology, 100(3), 426-432. https://doi.org/10.1037/a0022790

Wang, B., Theeuwes, J., \& Olivers, C. N. L. (2018). When shorter delays lead to worse memories: Task disruption makes visual working memory temporarily vulnerable to test interference. Journal of Experimental Psychology: Learning, Memory, and Cognition, 44(5), 722-733. https://doi.org/10.1037/xlm0000468

Wang, B., Theeuwes, J., \& Olivers, C. N. L. (2019). Momentary, Offset-Triggered DualTask Interference in Visual Working Memory. Journal of Cognition, 2(1), 38. https://doi.org/10.5334/joc.84

Wilken, P., \& Ma, W. J. (2004). A detection theory account of change detection. Journal of Vision, 4(12), 1120-1135. https://doi.org/10.1167/4.12.11

Williams, M., Hong, S. W., Kang, M.-S., Carlisle, N. B., \& Woodman, G. F. (2013). The benefit of forgetting. Psychonomic Bulletin \& Review, 20(2), 348-355. https://doi.org/10.3758/s13423-012-0354-3

Zhang, W., \& Luck, S. J. (2008). Discrete fixed-resolution representations in visual working memory. Nature, 453(7192), 233-235. https://doi.org/10.1038/nature06860 